\title{
Local exclusion and Lieb-Thirring inequalities for intermediate and fractional statistics
}

\author{
Douglas Lundholm ${ }^{a, *}$ and Jan Philip Solovej ${ }^{b, \dagger}$ \\ ${ }^{a}$ Institut Mittag-Leffler, Auravägen 17, SE-182 60 Djursholm, Sweden \\ ${ }^{b}$ Department of Mathematical Sciences, University of Copenhagen \\ Universitetsparken 5, DK-2100 Copenhagen $\varnothing$, Denmark
}

\begin{abstract}
In one and two spatial dimensions there is a logical possibility for identical quantum particles different from bosons and fermions, obeying intermediate or fractional (anyon) statistics. We consider applications of a recent Lieb-Thirring inequality for anyons in two dimensions, and derive new Lieb-Thirring inequalities for intermediate statistics in one dimension with implications for models of Lieb-Liniger and Calogero-Sutherland type. These inequalities follow from a local form of the exclusion principle valid for such generalized exchange statistics.

MSC2010: 81Q10, 81S05, 35P15, 46N50

Keywords: anyons, Calogero-Sutherland model, eigenvalue bounds, fractional statistics, Lieb-Liniger model, Lieb-Thirring inequality, selfadjoint extensions, stability
\end{abstract}

\section{Introduction}

Fundamental to many-body quantum mechanics is the notion of identical particles and associated particle statistics. Many of the remarkable quantum phenomena observed in nature are direct consequences of the fact that identical particles in three spatial dimensions are either bosons, obeying BoseEinstein statistics, or fermions, obeying Fermi-Dirac statistics. For example, the amplification of light in lasers by superposition of photons is possible because they are bosons, while the periodic table of elements arises from the sequential filling of atomic shells by electrons which are fermions. This binary classification has been known since the early days of quantum theory, and follows logically from the requirement that an $N$-body wave function $\psi\left(\boldsymbol{x}_{1}, \ldots, \boldsymbol{x}_{N}\right)$, modeled as a square-integrable complex-valued function 11 on

\footnotetext{
${ }^{*}$ Work partly done while visiting FIM, ETH Zürich. (e-mail: lundholm@math.ku.dk)

${ }^{\dagger}$ Work partly done while visiting Institut Mittag-Leffler. (e-mail: solovej@math.ku.dk)

${ }^{1}$ We will for simplicity always model particles as non-relativistic and scalar, i.e. without internal symmetries or spin.
} 
the $N$-particle configuration space $\left(\mathbb{R}^{3}\right)^{N}$, or alternatively as an element of the tensor product of one-particle spaces $\bigotimes^{N} L^{2}\left(\mathbb{R}^{3}\right)$, has to be either symmetric (corresponding to bosons) or antisymmetric (corresponding to fermions) under the action of permutations of the particle labels - which, if the particles are indistinguishable, must leave the observable probability $|\psi|^{2}$ invariant. However, it was discovered in the 1970-80's [58, 29, 22, 60] that this simple picture for identical particles and statistics is logically incomplete and should be replaced by a more general framework, which in three (or higher) space dimensions nicely reduces to the above binary picture, but in the one and two dimensional cases actually admits a wider range of possibilities. Identical particles classified via this generalized framework, but falling outside the usual boson/fermion classification, were said to obey intermediate or fractional statistics. Although elementary identical particles live in three space dimensions and hence must be either bosons or fermions, these more exotic one- and two-dimensional possibilities have recently become more than just a mathematical curiosity, with the advent of trapped bosonic condensates [27, 47] and quantum Hall physics [2, 28, 51], and thereby the discoveries of effective models of particles (or quasi-particles) which seem to obey these generalized rules for identical particles and statistics (see [6, 18, 26, 33, 45, 46, 50, 61] for reviews).

In two space dimensions the usual requirement, that the phase change of the wave function which arises from an interchange of two identical particles (or, in the more precise general framework, from a continuous simple interchange of two particles) needs to be either +1 (boson) or -1 (fermion), is no longer valid. This interchange phase is instead allowed to be any fixed unit complex number $e^{i \alpha \pi}$. The corresponding particles are called anyons, and are alternatively parameterized by the real-valued statistics parameter $\alpha \in(-1,1]$. A standard way to model such anyons is by means of bosons in $\mathbb{R}^{2}$ together with magnetic potentials $\boldsymbol{A}_{j}(\mathrm{x})$ of Aharonov-Bohm-type with strength $\alpha$ between every pair of particles (see (27) below), giving rise to the correct phase of the wave function as the particles interchange or encircle each other [29, 62]. The free kinetic energy operator for $N$ anyons is thus given by (see (27); we use the unit conventions $\hbar=m=1$ )

$$
\hat{T}_{\mathrm{A}}:=\frac{1}{2} \sum_{j=1}^{N} D_{j}^{2}, \quad D_{j}:=-i \nabla_{j}+\boldsymbol{A}_{j}(\mathrm{x}),
$$

and acting on completely symmetric wave functions $\psi \in \bigotimes_{\text {sym }}^{N} L^{2}\left(\mathbb{R}^{2}\right)$.

In one dimension, the above geometric picture of continuous interchanges of particles breaks down - particles have to collide in order to be interchanged. In a quantum mechanical context this necessitates the prescription of boundary conditions for the wave function at the collision points $r:=x_{j+1}-x_{j} \rightarrow 0^{+}$(i.e. at the boundary of the proper configuration space $X:=\left\{\mathrm{x} \in \mathbb{R}^{N}: x_{1}<x_{2}<\ldots<x_{N}\right\}$, in this case with codimension 
one). There are two ways in which one may arrive naturally at a set of boundary conditions. One approach [29, 1, which is generally referred to as Schrödinger-type quantization, starts out with the proper configuration space $X$ and imposes a general (Robin) boundary condition of the form $\partial_{r} \psi=\eta \psi$ at $r=0^{+}$, with $\eta \in \mathbb{R}$ an arbitrary but fixed statistics parameter. The case $\eta=0$ corresponds to bosons (Neumann b.c.) and $\eta=+\infty$ to fermions (Dirichlet b.c.). Such boundary conditions can, if $\psi$ is extended from $X$ to $\mathbb{R}^{N}$ and modeled symmetric about $r=0$, alternatively be encoded by a delta potential $2 \eta \delta(r)$, and the resulting model for $1 \mathrm{D}$ intermediate statistics, represented with a modified kinetic energy operator

$$
\hat{T}_{\mathrm{LL}}:=-\frac{1}{2} \sum_{j=1}^{N} \frac{\partial^{2}}{\partial x_{j}^{2}}+\sum_{1 \leq j<k \leq N} 2 \eta \delta\left(x_{k}-x_{j}\right)
$$

and acting on completely symmetric wave functions $\psi \in \bigotimes_{\text {sym }}^{N} L^{2}(\mathbb{R})$, is equivalent to the Lieb-Liniger model [34] for a gas of bosons on $\mathbb{R}$ with delta interactions.

Another, inequivalent but just as well-motivated, approach to 1D intermediate statistics is to consider Heisenberg-type quantization [30, 48, 24, 31] in the sense that one starts out with the classical phase space $\mathbb{R}^{N} \times \mathbb{R}^{N}$ and its observables (both should be symmetrized w.r.t. relabeling of particles) and considers representations of a corresponding algebra of operators. One finds, in the two-particle case, that the relevant representations are labeled by a continuous parameter $\alpha \in \mathbb{R}$, and that in the standard coordinate representation this amounts to imposing a boundary condition $\psi(r) \sim r^{\alpha}$ for the wave function as the relative coordinate $r \rightarrow 0^{+}$(this relation with boundary conditions is more complicated and will be elaborated in Section 2.1 below, but the restriction $\alpha \in(-1 / 2, \infty)$ is always required for squareintegrability). In this context bosons correspond to $\alpha=0$ and fermions to $\alpha=1$. Again, using symmetric $\psi$ the general boundary condition or choice of representation can equivalently be encoded by an interaction potential, of the form $\alpha(\alpha-1) / r^{2}$. The resulting model can be extended to the $N$-particle case and is equivalent to the homogeneous part of the Calogero-Sutherland model [8, 59], represented with a modified kinetic energy operator

$$
\hat{T}_{\mathrm{CS}}:=-\frac{1}{2} \sum_{j=1}^{N} \frac{\partial^{2}}{\partial x_{j}^{2}}+\sum_{1 \leq j<k \leq N} \frac{\alpha(\alpha-1)}{\left(x_{k}-x_{j}\right)^{2}},
$$

and again acting on completely symmetric wave functions $\psi \in \bigotimes_{\mathrm{sym}}^{N} L^{2}(\mathbb{R})$.

One may ask what properties these different types of identical particles possess. The fundamental characteristic property of fermions is that they obey Pauli's exclusion principle, i.e. the fact that the antisymmetry of the wave function implies that no two particles can occupy the same singleparticle state: $\varphi \wedge \varphi=0$ for any $\varphi \in L^{2}\left(\mathbb{R}^{d}\right)$. A powerful consequence of 
this is the Lieb-Thirring inequality [38, 39] (see also [37]) for the energy of $N$ (spinless) fermions in an external potential $V$ in $\mathbb{R}^{d}$, given by (again, with the conventions $\hbar=m=1$, and $\|\psi\|=1$ )

$$
\begin{aligned}
\int_{\mathbb{R}^{d N}} \sum_{j=1}^{N}\left(\frac{1}{2}\left|\nabla_{j} \psi\right|^{2}+\right. & \left.V\left(\boldsymbol{x}_{j}\right)|\psi|^{2}\right) d \mathrm{x} \\
& \geq-\sum_{k=0}^{N-1}\left|\lambda_{k}(h)\right| \geq-C_{d} \int_{\mathbb{R}^{d}}\left|V_{-}(\boldsymbol{x})\right|^{1+\frac{d}{2}} d \boldsymbol{x},
\end{aligned}
$$

where $V_{ \pm}:=(V \pm|V|) / 2$ and $C_{d}$ a positive constant. Here $\lambda_{k}(h)$ denote the negative eigenvalues (ordered by decreasing magnitude and with multiplicity) of the one-particle operator $h=-\frac{1}{2} \Delta+V(\boldsymbol{x})$. The first inequality expresses the Pauli exclusion principle while the second concerns the trace over the negative spectrum of $h$ and in some sense incorporates the uncertainty principle. In fact, the Lieb-Thirring inequality is equivalent to the kinetic energy inequality

$$
T_{0}:=\frac{1}{2} \int_{\mathbb{R}^{d N}} \sum_{j=1}^{N}\left|\nabla_{j} \psi\right|^{2} d \mathrm{x} \geq C_{d}^{\prime} \int_{\mathbb{R}^{d}} \rho(\boldsymbol{x})^{1+\frac{2}{d}} d \boldsymbol{x},
$$

with $C_{d}^{\prime}:=d\left(2 / C_{d}\right)^{2 / d} /(d+2)^{1+2 / d}$ another positive constant 2 , and $\rho$ the one-particle density function associated to $\psi$ :

$$
\rho(\boldsymbol{x}):=\sum_{j=1}^{N} \int_{\mathbb{R}^{d(N-1)}}\left|\psi\left(\boldsymbol{x}_{1}, \ldots, \boldsymbol{x}_{j-1}, \boldsymbol{x}, \boldsymbol{x}_{j+1}, \ldots, \boldsymbol{x}_{N}\right)\right|^{2} \prod_{k \neq j} d \boldsymbol{x}_{k} .
$$

The inequality (2) can be interpreted as a strong form of the uncertainty principle valid for fermions.

These inequalities need to be weakened in the case of weaker exclusion. E.g., in the situation that up to $q \in \mathbb{N}$ particles can occupy the same oneparticle state (sometimes referred to as Gentile intermediate statistics [19, 20]), the r.h.s. of (1) resp. (2) are to be multiplied by $q$ resp. $q^{-2 / d}$. For bosons there is no restriction on the number of particles in the same state; we can e.g. consider $\psi=\varphi_{0} \otimes \varphi_{0} \otimes \ldots \otimes \varphi_{0}$, where $\varphi_{0}$ is the ground state of $h$. Hence bosons can be accommodated by taking $q=N$, and the inequalities become trivial as $N \rightarrow \infty$. However, for intermediate and fractional statistics (in the above sense) the picture is more complicated. There have been partially successful attempts to relate also such many-body

\footnotetext{
${ }^{2}$ The currently best known value for $C_{d}^{\prime}$ is a factor $\left(3 / \pi^{2}\right)^{1 / d}$ smaller than its semiclassical value with $C_{d}=\frac{2^{d / 2}}{(2 \pi)^{d}} \int_{\mathbb{R}^{d}}\left(1-|\xi|^{2}\right)+d \xi[12$. It is conjectured that for $d=3$ the inequality (2) for fermions holds with exactly the semiclassical constant and the ThomasFermi expression on the r.h.s. [38].
} 
quantum states to one-particle states restricted by some exclusion principle [23, 25, 21], but so far no general picture has emerged. The difficulty has to do with the fact that these generalized exchange statistics are naturally modeled using interacting Hamiltonians, hence leaving the much simpler realm of single-particle operators and spaces.

In [42] we found that anyons in $\mathbb{R}^{2}$, with statistics parameter $\alpha=\mu / \nu$ an odd numerator fraction, satisfy a kinetic energy inequality of the form

$$
T_{\mathrm{A}}:=\frac{1}{2} \int_{\mathbb{R}^{2 N}} \sum_{j=1}^{N}\left|D_{j} \psi\right|^{2} d \mathrm{x} \geq \frac{1}{\nu^{2}} C_{\mathrm{A}} \int_{\mathbb{R}^{2}} \rho(\boldsymbol{x})^{2} d \boldsymbol{x},
$$

implying the Lieb-Thirring inequality

$$
\sum_{j=1}^{N} \int_{\mathbb{R}^{2 N}}\left(\frac{1}{2}\left|D_{j} \psi\right|^{2}+V\left(\boldsymbol{x}_{j}\right)|\psi|^{2}\right) d \mathrm{x} \geq-\nu^{2} C_{\mathrm{A}}^{\prime} \int_{\mathbb{R}^{2}}\left|V_{-}(\boldsymbol{x})\right|^{2} d \boldsymbol{x}
$$

for some positive constants $C_{\mathrm{A}}, C_{\mathrm{A}}^{\prime}$ (numerical estimates will be given below). For a general statistics parameter $\alpha \in \mathbb{R}$ and finite $N$ these inequalities hold with $1 / \nu^{2}$ replaced by $C_{\alpha, N}^{2}$, where

$$
C_{\alpha, N}:=\min _{p \in\{0,1, \ldots, N-2\}} \min _{q \in \mathbb{Z}}|(2 p+1) \alpha-2 q| .
$$

However, this expression tends to zero as $N \rightarrow \infty$, except when $\alpha=\mu / \nu$ is an odd numerator (reduced) fraction in which case $\lim _{N \rightarrow \infty} C_{\alpha, N}=1 / \nu$ (cp. Figure 2 in [42]). This, at first unexpected, difference between odd numerator fractions and even numerator and irrational $\alpha$ is further discussed in [43], where we also provide some arguments for this difference actually being natural.

In contrast to previous proofs of the standard Lieb-Thirring inequalities (11) and (2), the inequality (41) for anyons was proved using a local form of the uncertainty principle together with a local form of the exclusion principle, valid for anyons with general $\alpha$ and involving the constant $C_{\alpha, N}$ in (6) measuring the strength of exclusion. Our inspiration for this local approach to Lieb-Thirring inequalities, applicable to interacting systems, was the original proof of stability of ordinary fermionic matter [13] due to Dyson and Lenard (see also [14, 32]) which involved only local, comparatively weak, consequences of the Pauli principle for fermions (see Lemma 6 below).

\subsection{Main results}

In this work we consider further consequences of our results in [42 for anyons, and extend the above families of Lieb-Thirring-type inequalities to identical particles obeying intermediate exchange statistics in one dimension, in the above sense. We again start from a local form of the uncertainty 
principle together with a local exclusion principle of the form (cp. Lemma 6 below for fermions)

$$
\int_{[a, b]^{n}} \overline{\psi(\mathrm{x})}\left(\hat{T}_{\mathrm{LL} / \mathrm{CS}} \psi\right)(\mathrm{x}) d \mathrm{x} \geq(n-1) \frac{\xi_{\mathrm{LL} / \mathrm{CS}}^{2}}{|a-b|^{2}} \int_{[a, b]^{n}}|\psi(\mathrm{x})|^{2} d \mathrm{x}
$$

where in the Lieb-Liniger case $\xi_{\mathrm{LL}}$ is a measure of exclusion depending on $\eta$ times the length $|a-b|$ of a local interval, and in the Calogero-Sutherland case $\xi_{\mathrm{CS}}$ depends only on $\alpha$ (see Figure 1 resp. 2 below for their exact dependence).

Theorem 1. Let $\rho$ be the one-particle density (3) associated to a normalized completely symmetric wave function $\psi \in \bigotimes_{\mathrm{sym}}^{N} L^{2}(\mathbb{R})$ of $N \geq 2$ identical particles on the real line.

For the Lieb-Liniger case, with statistics parameter $\eta \geq 0$ and total kinetic energy $T_{\mathrm{LL}}=\left\langle\psi, \hat{T}_{\mathrm{LL}} \psi\right\rangle$, we have

$$
T_{\mathrm{LL}} \geq C_{\mathrm{LL}} \int_{\mathbb{R}} \xi_{\mathrm{LL}}\left(2 \eta / \rho^{*}(x)\right)^{2} \rho(x)^{3} d x
$$

where $\rho^{*}$ is the associated Hardy-Littlewood maximal function (see (70)), and $C_{\mathrm{LL}}$ is a universal constant satisfying $3 \cdot 10^{-5} \leq C_{\mathrm{LL}} \leq 2 / 3$.

For the Calogero-Sutherland case, with statistics parameter $\alpha \geq 1$ and total kinetic energy $T_{\mathrm{CS}}=\left\langle\psi, \hat{T}_{\mathrm{CS}} \psi\right\rangle$, and with its restriction to an arbitrary finite interval $Q \subseteq \mathbb{R}$ denoted $T_{\mathrm{CS}}^{Q}$ (see (47)), we have whenever $\int_{Q} \rho \geq 2$ that

$$
T_{\mathrm{CS}}^{Q} \geq C_{\mathrm{CS}} \xi_{\mathrm{CS}}(\alpha)^{2} \frac{\left(\int_{Q} \rho d x\right)^{3}}{|Q|^{2}}
$$

for a universal constant $1 / 32 \leq C_{\mathrm{CS}} \leq 2 / 3$.

In particular, if the density $\rho$ is confined to an interval of length $L$, then (9) implies for the energy per unit length

$$
T_{\mathrm{CS}} / L \geq C_{\mathrm{CS}} \xi_{\mathrm{CS}}(\alpha)^{2} \bar{\rho}^{3}
$$

where $\bar{\rho}:=N / L$ is the average density of particles. Note that $\xi_{\mathrm{CS}}(\alpha) \sim \alpha$ to leading order as $\alpha \rightarrow \infty$ (see Figure 2). We can compare this bound with Calogero [8] and Sutherland [59] (whose models are exactly solvable for certain choices of external potentials), where one finds for the ground state energy of a system confined to an interval

$$
T_{\mathrm{CS}} / L \rightarrow \frac{\pi^{2}}{6} \alpha^{2} \bar{\rho}^{3}
$$

in the thermodynamic limit $N, L \rightarrow \infty$ with fixed density $\bar{\rho}$. However, note that we do not need to assume a particular confinement potential for our 
bounds since they concern the kinetic energy $T_{\mathrm{CS}}$ alone. The possibility for considering more general external potentials in the Calogero-Sutherland models has also been discussed in the context of Thomas-Fermi theory; see [54, 55].

In the Lieb-Liniger case, the bound (8) implies that if $\rho^{*} \leq \gamma \bar{\rho}$ for some constant $\gamma>0$, i.e. if the density is sufficiently homogeneous, then

$$
T_{\mathrm{LL}} / L \geq C_{\mathrm{LL}} \xi_{\mathrm{LL}}(2 \eta /(\gamma \bar{\rho}))^{2} \bar{\rho}^{3} .
$$

We have $\xi_{\mathrm{LL}}(t) \sim \sqrt{t}$ for small $t$ and $\xi_{\mathrm{LL}}(t) \rightarrow \pi / 2$ as $t \rightarrow \infty$ (see Figure 11). Compare with Lieb and Liniger [34] (whose model is again exactly solvable in the absence of an external potential), where it is shown that in the thermodynamic limit the ground state energy satisfies

$$
T_{\mathrm{LL}} / L \rightarrow \frac{1}{2} e(2 \eta / \bar{\rho}) \bar{\rho}^{3},
$$

with an implicitly defined function $e$ s.t. $e(t) \sim t, t \ll 1, e(t) \rightarrow \frac{\pi^{2}}{3}, t \rightarrow \infty$. We can also compare with [36], where an energy functional of a form similar to the r.h.s. of (8) arises in the limit of tubular confinement of a threedimensional bosonic gas.

The methods we use to prove Theorem 1 are similar to those used for anyons in [42]. However, certain new technical complications arise in the onedimensional context, such as a local dependence of the strength of exclusion in the Lieb-Liniger case, and the possibility of arbitrarily strong exclusion in the Calogero-Sutherland case. This is the reason for the more complicated expressions (8) and (9) as compared to (21) and (41). There is certainly room for improvement in our bounds for the constants $C_{\mathrm{A} / \mathrm{LL} / \mathrm{CS}}$ (we mainly consider the forms of the inequalities and their dependence on the statistics parameters to be of conceptual interest), and for our methods we need to restrict to nonnegative statistics potentials, i.e. $\eta \geq 0$ resp. $\alpha \geq 1$. The intermediate Calogero-Sutherland case $\alpha \in(0,1)$ is certainly interesting (so is $\alpha \in(-1 / 2,0)$ ), but presents additional challenges and will not be addressed here.

In Section 2 we establish the context and notation of the paper, taking care to define the relevant operators properly. This is something we have not found been discussed in detail in the literature. In particular, we show that the formal definition of the kinetic energy form used for anyons in [42] is indeed the natural one. In Section 3 we recall the local exclusion principle for fermions and anyons, with an application of the latter to an explicit bound for the energy of the ideal anyon gas. We then introduce corresponding local principles of exclusion for 1D intermediate statistics. In Section 4 we deduce a local form of the uncertainty principle valid in arbitrary dimensions. These local bounds are then applied in Section 5 to prove Theorem 11. In the final section we consider applications of the Lieb-Thirring inequalities (4) and 
(5) for anyons to the old problem of many anyons confined in a harmonic oscillator potential, as well as the question of thermodynamic stability for a system of charged anyons and static particles interacting via 3D Coulomb potentials. We end with a discussion on the addition of external potentials for the Calogero-Sutherland case.

Acknowledgements. We thank Giovanni Felder, Jürg Fröhlich, Jens Hoppe, Edwin Langmann and Robert Seiringer for comments and discussions. Support from the Danish Council for Independent Research as well as from Institut Mittag-Leffler (Djursholm, Sweden) is gratefully acknowledged. D.L. would also like to thank IHÉS, FIM ETH Zurich, and the Isaac Newton Institute (EPSRC Grant EP/F005431/1) for support and hospitality via an EPDI fellowship, during which much of the present work was initiated.

\section{Identical particles in one and two dimensions}

We refer to the brief introduction in [42], the original references [58, 29, 22, 60], and the reviews [18, 61, 33, 45, 50, 26, 46], for a complete introduction to the general concepts of identical particles and exchange statistics in one and two dimensions. Here we will jump directly to the consequences of the general theory (for scalar particles) outlined in the introduction, and make precise our mathematical assumptions and notation.

As usual, we write $H^{k}(\Omega)$ for the Sobolev spaces of square-integrable functions on $\Omega \subseteq \mathbb{R}^{n}$ with square-integrable weak derivatives to order $k$. For a function space $\mathcal{F}$ we generally write $\mathcal{F}(X ; Y)$ to emphasize that the functions map $X \rightarrow Y$, suppressing the latter argument whenever $Y=\mathbb{C}$ or if otherwise understood from the context. The space of smooth and compactly supported functions on $\Omega$ is denoted by $C_{c}^{\infty}(\Omega)$ and its closure inside $H^{k}(\Omega)$ by $H_{0}^{k}(\Omega)$. Given a space $\mathcal{F}$ of functions on the (traditional) $N$-particle configuration space $X=\left(\mathbb{R}^{d}\right)^{N}$, the subspace of functions which are completely symmetric resp. antisymmetric w.r.t. particle permutations will be denoted $\mathcal{F}_{\text {sym }}$ resp. $\mathcal{F}_{\text {asym }}$. The domain of an operator $A$ is denoted by $\mathscr{D}(A)$.

\section{$2.1 \quad$ One dimension}

Depending on which approach one takes to quantization [29, 30, 48, 1, 24, 31, 25, 45], identical particles in 1D can be modeled as bosons, i.e. wave functions symmetric under the flip $r \mapsto-r$ of any two relative particle coordinates $r:=x_{j}-x_{k}$, together with a local interaction potential, singular at $r=0$ and either of the form $\delta_{0}(r)$ or $1 / r^{2}$. We write (cp. e.g. [45])

$$
V_{\mathrm{LL}}(r):=2 \eta \delta_{0}(r), \quad V_{\mathrm{CS}}(r):=\frac{\alpha(\alpha-1)}{r^{2}},
$$


with statistics parameters $\eta, \alpha \in \mathbb{R}$, for the corresponding cases of Schrödinger- resp. Heisenberg-type quantization. These statistics potentials, which coincide with the interaction potentials of the Lieb-Liniger resp. CalogeroSutherland models, should correspond to the choices of boundary conditions (in a sense to be made precise below)

$$
\frac{\partial \psi}{\partial r}=\eta \psi, \quad \text { at } r=0^{+}
$$

resp.

$$
\psi(r) \sim r^{\alpha}, \quad r \rightarrow 0^{+},
$$

for the wave function $\psi$ at the boundary $r=0$ of the configuration space. Note that in this sense $\eta=0$ resp. $\alpha=0$ represent bosons, while $\eta=+\infty$ resp. $\alpha=1$ represent fermions (in the bosonic representation, i.e. after factoring out the sign of the permutation). Suggested by such pairwise boundary conditions, one may define (cp. e.g. 45) the total kinetic energy for a normalized wave function $\psi \in L_{\text {sym }}^{2}:=\bigotimes_{\text {sym }}^{N} L^{2}(\mathbb{R})$ of $N$ identical particles on $\mathbb{R}$ to be $T_{\mathrm{LL} / \mathrm{CS}}:=\int_{\mathbb{R}^{N}} T_{\mathrm{LL} / \mathrm{CS}}^{N}(\psi ; \mathrm{x}) d \mathrm{x}$ where

$$
T_{\mathrm{LL} / \mathrm{CS}}^{N}(\psi ; \mathrm{x}):=\frac{1}{2} \sum_{j=1}^{N}\left|\partial_{j} \psi\right|^{2}+\sum_{1 \leq j<k \leq N} V_{\mathrm{LL} / \mathrm{CS}}\left(x_{j}-x_{k}\right)|\psi|^{2},
$$

with a corresponding kinetic energy operator

$$
\hat{T}_{\mathrm{LL} / \mathrm{CS}}=\hat{T}_{0}+\hat{V}_{\mathrm{LL} / \mathrm{CS}}:=-\frac{1}{2} \sum_{j=1}^{N} \frac{\partial^{2}}{\partial x_{j}^{2}}+\sum_{1 \leq j<k \leq N} V_{\mathrm{LL} / \mathrm{CS}}\left(x_{j}-x_{k}\right) .
$$

Formally, we need to specify domains $\mathscr{D}_{\mathrm{LL} / \mathrm{CS}}$ resp. $\hat{\mathscr{D}}_{\mathrm{LL} / \mathrm{CS}}$ for the quadratic forms $\psi \mapsto T_{\mathrm{LL} / \mathrm{CS}}(\psi)$ and operators $\hat{T}_{\mathrm{LL} / \mathrm{CS}}$ so that they are closed, respectively self-adjoint. Let (for arbitrary dimension $d$ )

$$
\triangle:=\left\{\mathrm{x} \in\left(\mathbb{R}^{d}\right)^{N}: \exists j \neq k \text { s.t. } \boldsymbol{x}_{j}=\boldsymbol{x}_{k}\right\}
$$

denote the diagonal set where any two particles meet, i.e. where the statistics potentials $V_{\mathrm{LL} / \mathrm{CS}}$ are singular. In the Lieb-Liniger case,

$$
T_{\mathrm{LL}}(\psi)=\frac{1}{2} \int_{\mathbb{R}^{N}}|\nabla \psi|^{2} d \mathrm{x}+2 \eta \int_{\triangle}|\psi|^{2} d \Sigma_{\triangle}
$$

(with $\Sigma_{\triangle}$ the Euclidean measure on $\triangle$ ) defines a closed and semibounded quadratic form on the domain $\mathscr{D}_{\mathrm{LL}}:=H^{1}\left(\mathbb{R}^{N}\right) \cap L_{\text {sym }}^{2}$, and hence also defines an associated self-adjoint operator $\hat{T}_{\mathrm{LL}}$ on $L_{\mathrm{sym}}^{2}$. Its domain $\hat{\mathscr{D}}_{\mathrm{LL}}$ is s.t. $\psi \in$ $\hat{\mathscr{D}}_{\mathrm{LL}}$ is twice (weakly) differentiable on $\mathbb{R}^{N} \backslash \triangle$, continuous at the diagonals $\triangle$, and satisfies (cp. also [34])

$$
\left.\left(\frac{\partial}{\partial x_{j}}-\frac{\partial}{\partial x_{k}}\right) \psi\right|_{x_{j}=x_{k}^{+}}-\left.\left(\frac{\partial}{\partial x_{j}}-\frac{\partial}{\partial x_{k}}\right) \psi\right|_{x_{j}=x_{k}^{-}}=\left.4 \eta \psi\right|_{x_{j}=x_{k}} .
$$


In the Calogero-Sutherland case, there is a complication in the choice of domains associated with the symmetry $\alpha \mapsto 1-\alpha$. We therefore begin by considering the quadratic form

$$
T_{\mathrm{CS}}(\psi)=\frac{1}{2} \int_{\mathbb{R}^{N}}|\nabla \psi|^{2} d \mathrm{x}+\alpha(\alpha-1) \int_{\mathbb{R}^{N}} \sum_{j<k} \frac{|\psi|^{2}}{\left|x_{j}-x_{k}\right|^{2}} d \mathrm{x}
$$

initially on the nice space of functions $\psi \in C_{c}^{\infty}\left(\mathbb{R}^{N} \backslash \triangle\right) \cap L_{\text {sym. }}^{2}$ We can then use the substitution $\psi=f^{\alpha} \phi, f(\mathrm{x}):=\prod_{j<k}\left|x_{j}-x_{k}\right|$, and a partial integration to write (see e.g. [41])

$$
\int_{\mathbb{R}^{N}}|\nabla \psi|^{2} d \mathrm{x}=\int_{\mathbb{R}^{N}}\left(\alpha(1-\alpha) \frac{|\nabla f|^{2}}{f^{2}}+\alpha \frac{-\Delta f}{f}\right)|\psi|^{2} d \mathrm{x}+\int_{\mathbb{R}^{N}}|\nabla \phi|^{2} f^{2 \alpha} d \mathrm{x},
$$

where $\Delta f=0$ on $\mathbb{R}^{N} \backslash \triangle$ and $|\nabla f| / f^{2}=2 \sum_{j<k}\left|x_{j}-x_{k}\right|^{-2}$. Hence we have the representation $T_{\mathrm{CS}}(\psi)=\frac{1}{2}\left\|Q_{\alpha} \psi\right\|^{2} \geq 0$ for such $\psi$ and all $\alpha \in \mathbb{R}$, where $Q_{\alpha}$ denotes the vector-valued differential expression (cp. also [49])

$$
Q_{\alpha}:=f^{\alpha} \nabla f^{-\alpha}=\nabla-\alpha W, \quad W_{j}(\mathrm{x}):=\sum_{k \neq j}\left(x_{j}-x_{k}\right)^{-1} .
$$

Now, consider $Q_{\alpha}: L^{2}\left(\mathbb{R}^{N}\right) \rightarrow \mathcal{D}^{\prime}\left(\mathbb{R}^{N} \backslash \triangle ; \mathbb{C}^{N}\right)$ as a distribution-valued operator (note that since $W \notin L_{\text {loc }}^{1}\left(\mathbb{R}^{N}\right)$ we need to be careful and remove the diagonals), and define $T_{\mathrm{CS}}$ with domain $\mathscr{D}_{\mathrm{CS}}:=\mathscr{D}\left(Q_{\alpha}^{\max }\right)$ to be the quadratic form $T_{\mathrm{CS}}(\psi):=\frac{1}{2}\left\|Q_{\alpha}^{\max } \psi\right\|^{2}$ associated to the maximal extension of the operator $Q_{\alpha}$ on $L_{\text {sym }}^{2}$, i.e.

$$
\mathscr{D}\left(Q_{\alpha}^{\max }\right):=\left\{\psi \in L_{\text {sym }}^{2}: Q_{\alpha} \psi \in L^{2}\left(\mathbb{R}^{N} ; \mathbb{C}^{N}\right)\right\},
$$

with the identification $L^{2}\left(\mathbb{R}^{N} ; \mathbb{C}^{N}\right)=L^{2}\left(\mathbb{R}^{N} \backslash \triangle ; \mathbb{C}^{N}\right)$. Note that this definition of $T_{\mathrm{CS}}$ does not exclude the boundary behavior (16) for $\alpha>-1 / 2$, taking e.g. $\psi(\mathrm{x})=f^{\alpha}(\mathrm{x}) e^{-|\mathrm{x}|^{2}} \in \mathscr{D}_{\mathrm{CS}}$, and that for $\alpha=0, \mathscr{D}_{\mathrm{CS}}=H_{\mathrm{sym}}^{1}\left(\mathbb{R}^{N} \backslash \triangle\right)=$ $H_{\text {sym }}^{1}\left(\mathbb{R}^{N}\right)$ (note that the symmetry requirement is important here). The self-adjoint operator $\hat{T}_{\mathrm{CS}}:=\frac{1}{2}\left(Q_{\alpha}^{\max }\right)^{*} Q_{\alpha}^{\max }$ is then defined with domain

$$
\hat{\mathscr{D}}_{\mathrm{CS}}:=\left\{\psi \in \mathscr{D}_{\mathrm{CS}}: Q_{\alpha} \psi \in \mathscr{D}\left(\left(Q_{\alpha}^{\max }\right)^{*}\right)\right\} \text {. }
$$

Another option would be to define $2 \hat{T}_{\mathrm{CS}}$ to be the Friedrichs extension, i.e. $\left(Q_{\alpha}^{\min }\right)^{*} Q_{\alpha}^{\min }$, associated to the minimally extended operator $Q_{\alpha}^{\min }$, its domain $\mathscr{D}\left(Q_{\alpha}^{\min }\right)$ being the closure of $C_{c}^{\infty}\left(\mathbb{R}^{N} \backslash \triangle\right)$ inside $L_{\text {sym }}^{2}$ w.r.t. the form $\psi \mapsto\left\|Q_{\alpha} \psi\right\|^{2}$. In this case we can explicitly characterize its domain as $\mathscr{D}\left(Q_{\alpha}^{\min }\right)=H_{0, \text { sym }}^{1}\left(\mathbb{R}^{N} \backslash \triangle\right)$ for $\alpha \neq 1 / 2$. This follows because for $\psi \in$ $C_{c}^{\infty}\left(\mathbb{R}^{N} \backslash \Delta\right.$ ) the identity (23) for $\alpha \neq 1 / 2$ and for $\alpha=1 / 2$ (the Hardy inequality) implies

$$
c_{1}\|\nabla \psi\| \leq\left\|Q_{\alpha} \psi\right\| \leq c_{2}\|\nabla \psi\|
$$


for some constants $c_{1}, c_{2}>0$ (depending on $\alpha \neq 1 / 2$ ), and hence taking the closure w.r.t. the $H^{1}$-form is the same as w.r.t. the $Q_{\alpha}$-form.

On the other hand, the following shows that the two extensions $Q_{\alpha}^{\min }$ and $Q_{\alpha}^{\max }$ are the same for sufficiently large $\alpha$ (while they are in general not for small $\alpha$, such as $\alpha=0$; see also [7, 4, 16]):

Theorem 2. For $\alpha \geq 1$ we have $Q_{\alpha}^{\min }=Q_{\alpha}^{\max }$, with domain

$$
\mathscr{D}_{\mathrm{CS}}=H_{0, \mathrm{sym}}^{1}\left(\mathbb{R}^{N} \backslash \triangle\right) \text {, }
$$

and hence the operator $\hat{T}_{\mathrm{CS}}=\frac{1}{2}\left(Q_{\alpha}^{\max }\right)^{*} Q_{\alpha}^{\max }$ is equal to the Friedrichs extension $\hat{T}_{\mathrm{CS}}=\frac{1}{2}\left(Q_{\alpha}^{\min }\right)^{*} Q_{\alpha}^{\min }$. In the case $N=2$ we have for all $\alpha>1 / 2$ that $Q_{\alpha}^{\min }=Q_{\alpha}^{\max }$ with $\mathscr{D}_{\mathrm{CS}}=H_{0, \mathrm{sym}}^{1}\left(\mathbb{R}^{2} \backslash \triangle\right)$.

Proof. We claim that, for $\alpha \geq 1, \psi \in \mathscr{D}_{\mathrm{CS}}$ must satisfy $\psi \in H^{1}\left(\mathbb{R}^{N}\right)$ as well as

$$
\int_{\mathbb{R}^{N}} \frac{|\psi|^{2}}{\left(x_{j}-x_{k}\right)^{2}} d \mathrm{x}<\infty \quad \text { for all } j \neq k,
$$

from which approximability in $H_{0}^{1}\left(\mathbb{R}^{N} \backslash \triangle\right) \cap L_{\text {sym }}^{2}$ follows by taking $\psi_{\varepsilon}(\mathrm{x}):=$ $\prod_{j<k} \phi_{\varepsilon}\left(x_{j}-x_{k}\right) \psi(\mathrm{x})$, where $\phi_{\varepsilon}(x):=\phi(x / \varepsilon)$ and $\phi \in C^{\infty}(\mathbb{R} ;[0,1])$ symmetric and identically zero for $|x| \leq 1$ and one for $|x| \geq 2$. Then $\psi_{\varepsilon} \in \mathscr{D}_{\mathrm{CS}}$ and

$$
Q_{\alpha} \psi_{\varepsilon}-Q_{\alpha} \psi=\left(\prod \phi_{\varepsilon}-1\right) Q_{\alpha} \psi+\left(\nabla \prod \phi_{\varepsilon}\right) \psi,
$$

where $\left\|\left(\prod \phi_{\varepsilon}-1\right) \psi\right\| \rightarrow 0$ and $\left\|\left(\prod \phi_{\varepsilon}-1\right) Q_{\alpha} \psi\right\| \rightarrow 0$ as $\varepsilon \rightarrow 0$ by dominated convergence, and for the second term we have by (24)

$$
\begin{aligned}
& \left\|\phi_{\varepsilon}^{\prime}\left(x_{j}-x_{k}\right) \psi\right\|^{2}=\int_{\mathbb{R}^{N}} \frac{\left|\phi^{\prime}\left(\left(x_{j}-x_{k}\right) / \varepsilon\right)\right|^{2}}{\varepsilon^{2}}|\psi|^{2} d \mathrm{x} \\
& \quad \leq C \int_{\varepsilon<\left|x_{j}-x_{k}\right|<2 \varepsilon} \frac{|\psi|^{2}}{\left(x_{j}-x_{k}\right)^{2}} d \mathrm{x} \rightarrow 0 .
\end{aligned}
$$

Hence, $\left\|Q_{\alpha}\left(\psi_{\varepsilon}-\psi\right)\right\| \rightarrow 0$ as $\varepsilon \rightarrow 0$.

To prove the above claim, consider the pointwise a.e. in $\mathbb{R}^{N} \backslash \triangle$ identity

$$
\begin{aligned}
\left|Q_{\alpha} \psi\right|^{2}=|\nabla \psi|^{2}+ & \alpha^{2} W \cdot W|\psi|^{2}-\alpha W \cdot((\nabla \bar{\psi}) \psi+\bar{\psi} \nabla \psi) \\
=|\nabla \psi|^{2}+\alpha^{2} W^{2}|\psi|^{2}-\alpha W \cdot \nabla|\psi|^{2} & =|\nabla \psi|^{2}+\alpha(\alpha-1) W^{2}|\psi|^{2}-\alpha \nabla \cdot\left(W|\psi|^{2}\right) \\
& =\mid
\end{aligned}
$$

where in the second step we used that $\psi \in H_{\text {loc }}^{1}\left(\mathbb{R}^{N} \backslash \triangle\right)$ since $W \psi \in$ $L_{\text {loc }}^{2}\left(\mathbb{R}^{N} \backslash \triangle\right.$ ) and $Q_{\alpha} \psi=\nabla \psi-\alpha W \psi \in L^{2}\left(\mathbb{R}^{N}\right)$ (note that then $\partial_{j}|\psi|^{2}-$ $\left(\partial_{j} \bar{\psi}\right) \psi-\bar{\psi}\left(\partial_{j} \psi\right)=0$ in $\mathcal{D}^{\prime}\left(\mathbb{R}^{N} \backslash \triangle\right)$ and $\left.\nabla|\psi|^{2} \in L_{\mathrm{loc}}^{1}\left(\mathbb{R}^{N} \backslash \triangle\right)\right)$, and in the 
third step we have $-\nabla \cdot W=W^{2}=|\nabla f| / f^{2}=2 \sum_{j<k}\left|x_{j}-x_{k}\right|^{-2}$. Now, for any $\varepsilon \geq 0$, let us define the wedge-shaped set

$$
\begin{aligned}
\Gamma_{\varepsilon}:=\left\{\mathrm{x} \in \mathbb{R}^{N}: x_{1}+(N-1) \varepsilon<x_{2}+\right. & \left.(N-2) \varepsilon<\ldots<x_{N-1}+\varepsilon<x_{N}\right\} \\
& =\bigcap_{j=1}^{N-1}\left\{\mathrm{x} \in \mathbb{R}^{N}: x_{j}+\varepsilon<x_{j+1}\right\},
\end{aligned}
$$

with piecewise flat boundary $\partial \Gamma_{\varepsilon}=\bigcup_{j=1}^{N-1}\left(\partial \Gamma_{\varepsilon}\right)_{j, j+1}$,

$$
\left(\partial \Gamma_{\varepsilon}\right)_{j, j+1}:=\left\{\mathrm{x} \in \mathbb{R}^{N}: x_{j}+\varepsilon=x_{j+1} \text { and } x_{k}+\varepsilon \leq x_{k+1} \text { for } k \neq j\right\} .
$$

By (25) and the divergence theorem (see e.g. Theorem 6.9 in [35]) we have for $\varepsilon>0$

$$
\int_{\Gamma_{\varepsilon}}\left|Q_{\alpha} \psi\right|^{2} d \mathrm{x}=\int_{\Gamma_{\varepsilon}}\left(|\nabla \psi|^{2}+\alpha(\alpha-1) W^{2}|\psi|^{2}\right) d \mathrm{x}-\alpha \int_{\partial \Gamma_{\varepsilon}} \hat{n} \cdot W|\psi|^{2} d \mathrm{x} .
$$

On the boundary component $\left(\partial \Gamma_{\varepsilon}\right)_{j, j+1}$, the unit outward normal is $\hat{n}=$ $\left(e_{j}-e_{j+1}\right) / \sqrt{2}$, and we obtain pointwise

$$
\begin{gathered}
\sqrt{2} \hat{n} \cdot W=W_{j}-W_{j+1} \\
=2\left(x_{j}-x_{j+1}\right)^{-1}+\sum_{\substack{k=1 \\
k \neq j, j+1}}^{N}\left(\left(x_{j}-x_{k}\right)^{-1}-\left(x_{j+1}-x_{k}\right)^{-1}\right) \\
=-2 \varepsilon^{-1}+\sum_{\substack{k \neq j, j+1\\
}} \varepsilon\left(x_{j}-x_{k}\right)^{-1}\left(x_{j}-x_{k}+\varepsilon\right)^{-1} \\
=-2 \varepsilon^{-1}+\sum_{k<j} \varepsilon\left(x_{j}-x_{k}\right)^{-1}\left(x_{j}-x_{k}+\varepsilon\right)^{-1}+\sum_{k>j+1} \varepsilon\left(x_{k}-x_{j}\right)^{-1}\left(x_{k}-x_{j}-\varepsilon\right)^{-1} .
\end{gathered}
$$

For $N=2$ we then have $-\alpha \hat{n} \cdot W=\frac{\alpha \varepsilon^{-1}}{\sqrt{2}} 2$, while for $N>2$ the two additional positive sums above are bounded by

$$
\sum_{k=1}^{j-1} \varepsilon((j-k) \varepsilon)^{-1}((j-k) \varepsilon+\varepsilon)^{-1}=\varepsilon^{-1} \sum_{k=1}^{j-1} \frac{1}{k(k+1)}=\varepsilon^{-1} \frac{j-1}{j},
$$

respectively

$$
\sum_{k=j+2}^{N} \varepsilon((k-j) \varepsilon)^{-1}((k-j) \varepsilon-\varepsilon)^{-1}=\varepsilon^{-1} \sum_{k=1}^{N-j-1} \frac{1}{k(k+1)}=\varepsilon^{-1} \frac{N-j-1}{N-j},
$$

and hence

$$
-\alpha \hat{n} \cdot W \geq \frac{\alpha \varepsilon^{-1}}{\sqrt{2}}\left(2-\frac{j-1}{j}-\frac{N-j-1}{N-j}\right)=\frac{\alpha \varepsilon^{-1}}{\sqrt{2}} \frac{N}{j(N-j)} \geq \frac{\alpha \varepsilon^{-1}}{\sqrt{2}} \frac{4}{N},
$$


which is strictly positive for all $N>2$. Hence, since all terms in the r.h.s. of (26) are nonnegative for $\alpha \geq 1$, and the 1.h.s. remains finite as $\varepsilon \rightarrow 0$, we must have $\nabla \psi, W \psi \in L^{2}\left(\Gamma_{0}\right)$ (also in the case $\alpha=1$ we find $\nabla \psi \in L^{2}\left(\Gamma_{0}\right)$ and therefore $W \psi \in L^{2}\left(\Gamma_{0}\right)$ since $\left.Q_{\alpha} \psi \in L^{2}\left(\Gamma_{0}\right)\right)$. By symmetry of $\psi$, this also holds on the remaining parts of $\mathbb{R}^{N} \backslash \triangle$ obtained by permutation of the coordinates, and this would then prove the claim if we only knew that $\nabla \psi \in \mathcal{D}^{\prime}\left(\mathbb{R}^{N}\right)$ is really a function on all of $\mathbb{R}^{N}$ and not also having some singular component supported on $\triangle$. The support must furthermore be of codimension one (cp. Lemma 3 below). But this would require a codimension one discontinuity of $\psi$ at one of the boundary components $\left(\partial \Gamma_{0}\right)_{j, j+1}$, which is in contradiction with both $W \psi \in L^{2}\left(\mathbb{R}^{N}\right)$ and the symmetry of $\psi$. This proves the first part of the theorem.

It remains to prove the stronger statement of the theorem for the special case $N=2$ and $\alpha>1 / 2$ (this was also proved differently in [7], in a oneparticle form). We start by showing that $\left|x_{1}-x_{2}\right|^{-\frac{1}{2}} \psi$ is (transversally) uniformly bounded if $\alpha>1 / 2$. Note that for $x_{1}<x_{2}$,

$$
\begin{gathered}
\left(x_{2}-x_{1}\right)^{-\alpha} \psi(\mathrm{x})=-\int_{0}^{\infty} \frac{d}{d t}\left(\left(x_{2}-x_{1}+2 t\right)^{-\alpha} \psi\left(x_{1}-t, x_{2}+t\right)\right) d t \\
=-\int_{0}^{\infty}\left(x_{2}-x_{1}+2 t\right)^{-\alpha}\left(\left(\partial_{2}-\partial_{1}\right) \psi\left(x_{1}-t, x_{2}+t\right)\right. \\
\left.\quad-2 \alpha\left(x_{2}-x_{1}+2 t\right)^{-1} \psi\left(x_{1}-t, x_{2}+t\right)\right) d t \\
=-\int_{0}^{\infty}\left(x_{2}-x_{1}+2 t\right)^{-\alpha}\left(\left(Q_{\alpha, 2}-Q_{\alpha, 1}\right) \psi\right)\left(x_{1}-t, x_{2}+t\right) d t \\
\leq\left.\left.\left|\int_{0}^{\infty}\left(x_{2}-x_{1}+2 t\right)^{-2 \alpha} d t\right|^{\frac{1}{2}}\left|\int_{0}^{\infty}\right|\left(Q_{\alpha, 2}-Q_{\alpha, 1}\right) \psi\right|^{2}\left(x_{1}-t, x_{2}+t\right) d t\right|^{\frac{1}{2}} \\
\leq\left(\frac{\left(x_{2}-x_{1}\right)^{1-2 \alpha}}{4 \alpha-2}\right)^{\frac{1}{2}}\left\|\left(Q_{\alpha, 2}-Q_{\alpha, 1}\right) \psi\right\|_{r}
\end{gathered}
$$

where we used Cauchy-Schwarz, and $\|\cdot\|_{r}$ denotes the $L^{2}$-norm w.r.t. the transversal variable $r=x_{2}-x_{1}$ (but still depending on the longitudinal center-of-mass $\left.R=\left(x_{1}+x_{2}\right) / 2\right)$. Similarly for $x_{1}>x_{2}$, we have $|r|^{-\frac{1}{2}} \psi(\mathrm{x}) \leq$ $\left\|\left(Q_{\alpha, 2}-Q_{\alpha, 1}\right) \psi\right\|_{r} / \sqrt{4 \alpha-2}$. Taking $\psi_{\varepsilon}(\mathrm{x}):=\varphi_{\varepsilon}\left(x_{1}-x_{2}\right) \psi(\mathrm{x})$, with $\varphi_{\varepsilon}$ a logarithmic cut-off function defined as in the proof of Lemma 3 below, we have

$$
Q_{\alpha} \psi_{\varepsilon}-Q_{\alpha} \psi=\left(\varphi_{\varepsilon}-1\right) Q_{\alpha} \psi \pm \varphi_{\varepsilon}^{\prime} \psi
$$

The norm-squared of the last term is

$$
\begin{aligned}
\varepsilon^{2} \int_{R} \int_{r} \frac{\left|\varphi^{\prime}(\varepsilon \ln |r|)\right|^{2}}{r^{2}}|\psi|^{2} d r d R \leq C \varepsilon \int_{R} \|\left(Q_{\alpha, 2}\right. & \left.-Q_{\alpha, 1}\right) \psi \|_{r}^{2} d R \\
& =C \varepsilon\left\|\left(Q_{\alpha, 2}-Q_{\alpha, 1}\right) \psi\right\|^{2}
\end{aligned}
$$


which shows that $\left\|Q_{\alpha}\left(\psi_{\varepsilon}-\psi\right)\right\| \rightarrow 0$ as $\varepsilon \rightarrow 0$, with $\psi_{\varepsilon} \in H_{0, \text { sym }}^{1}\left(\mathbb{R}^{2} \backslash \triangle\right)$.

We will in the following always assume $\eta \geq 0$ resp. $\alpha \geq 1$ in order for the statistics potentials $V_{\mathrm{LL}}$ resp. $V_{\mathrm{CS}}$ to be nonnegative, and can hence work with the Friedrichs extension for $\hat{T}_{\mathrm{CS}}$. To emphasize the dependence on the statistics parameters, the corresponding domains will be denoted $\hat{\mathscr{D}}_{\mathrm{LL}}^{\eta} \subseteq \mathscr{D}_{\mathrm{LL}}^{\eta}$ resp. $\hat{\mathscr{D}}_{\mathrm{CS}}^{\alpha} \subseteq \mathscr{D}_{\mathrm{CS}}^{\alpha}$. It is well-known that in the exactly solvable Calogero-Sutherland models, involving the operator $\hat{T}_{\mathrm{CS}}$, the eigenfunctions satisfy the boundary condition (16) (see [8, 59]). However, for large $\alpha$ this requirement is too restrictive, since e.g. $\psi(\mathrm{x})=f^{2}(\mathrm{x}) e^{-|\mathrm{x}|^{2}}$ is both in $\mathscr{D}_{\mathrm{CS}}^{\alpha}$ and $\hat{\mathscr{D}}_{\mathrm{CS}}^{\alpha}$, even for $\alpha>2$. The explicit dependence on $\alpha$ of the domains $\mathscr{D}_{\mathrm{CS}}^{\alpha}$ and $\hat{\mathscr{D}}_{\mathrm{CS}}^{\alpha}$ for the operators appearing in the two-particle case $N=2$ has been thoroughly investigated in [7]. Also the extension theory for the $N=3$ case has been considered in detail in [16]. The complete behavior in the manyparticle case for $\alpha \in(-1 / 2,1)$ remains, to the best of our knowledge, an interesting open problem.

\subsection{Two dimensions}

(Abelian) anyons in $\mathbb{R}^{2}$ comprise a continuous family of identical particles, characterized by a one-dimensional unitary representation of the braid group $B_{N}$, i.e. a complex phase $e^{i \alpha \pi} \in U(1)$, or a real statistics parameter $\alpha \in \mathbb{R}$ (modulo 2). For $\alpha=0$ such particles are bosons while for $\alpha=1$ they are fermions. For general $\alpha$, one can model quantum mechanical wave functions $\psi$ of $N$ such anyons by means of bosonic wave functions on $\mathbb{R}^{2}$, i.e. completely symmetric functions in $L^{2}\left(\left(\mathbb{R}^{2}\right)^{N}\right)$, together with magnetic interaction potentials $\boldsymbol{A}_{j}:=\alpha \sum_{k \neq j}\left(\boldsymbol{x}_{j}-\boldsymbol{x}_{k}\right)^{-1} I$ of topological type between all particles. Here $\boldsymbol{x} I$ denotes the $90^{\circ}$ counter-clockwise rotation of the vector $\boldsymbol{x} \in \mathbb{R}^{2}$, and $\boldsymbol{x}^{-1}:=\boldsymbol{x} /|\boldsymbol{x}|^{2}$. Hence, the total kinetic energy for $\psi$ is given by $T_{\mathrm{A}}:=\int_{\mathbb{R}^{2 N}} T_{\mathrm{A}}^{N}(\psi ; \mathrm{x}) d \mathrm{x}$, where

$T_{\mathrm{A}}^{N}(\psi ; \mathrm{x}):=\frac{1}{2} \sum_{j=1}^{N}\left|D_{j} \psi\right|^{2}, \quad D_{j}:=-i \nabla_{j}+\boldsymbol{A}_{j}=-i \nabla_{\boldsymbol{x}_{j}}+\alpha \sum_{\substack{k=1 \\ k \neq j}}^{N}\left(\boldsymbol{x}_{j}-\boldsymbol{x}_{k}\right)^{-1} I$,

corresponding to a kinetic energy operator

$$
\hat{T}_{\mathrm{A}}:=\frac{1}{2} \sum_{j=1}^{N} D_{j} \cdot D_{j}
$$

There is also here a question on how this operator and quadratic form should be formally defined since $\boldsymbol{A}_{j}$ (x) diverges badly as $\boldsymbol{x}_{k} \rightarrow \boldsymbol{x}_{j}$. In [42] we defined the operator $\hat{T}_{\mathrm{A}}$ in (28) with a closed quadratic form $T_{\mathrm{A}}$ as the Friedrichs extension initially defined on $C_{c}^{\infty}\left(\mathbb{R}^{2 N} \backslash \triangle\right) \cap L_{\text {sym }}^{2}$. This might seem to imply 
a mild hard-core requirement for the wave function for $\alpha \neq 0$ (cp. e.g. 40, 3, where a stronger form of hard-core requirement for anyons was imposed). Let us first observe explicitly that this is not the case whenever $\alpha \in 2 \mathbb{Z}$ (respecting the periodicity of the statistics parameter), and then show for general $\alpha \in \mathbb{R}$ that this definition is truly non-restrictive in the sense that it coincides with that of the maximal extension for the quadratic form $T_{\mathrm{A}}$. In other words, if we insist on the kinetic energy form (27) being finite, then we necessarily have this self-adjoint extension.

We begin by defining the magnetic derivative as the distribution-valued operator

$$
D: L^{2}\left(\mathbb{R}^{2 N}\right) \rightarrow \mathcal{D}^{\prime}\left(\mathbb{R}^{2 N} \backslash \triangle ; \mathbb{C}^{2 N}\right), \quad \psi \mapsto\left(D_{j} \psi\right)_{j=1}^{N},
$$

where $D_{j}$ are the $\mathbb{C}^{2}$-vector-valued differential expressions in (27). Note that we again need to consider this as a distribution strictly outside the diagonals since $\boldsymbol{A}_{j} \notin L_{\text {loc }}^{2}\left(\mathbb{R}^{2 N}\right)$ and hence $\boldsymbol{A}_{j} \psi \notin L_{\text {loc }}^{1}\left(\mathbb{R}^{2 N}\right)$, although $\boldsymbol{A}_{j} \in C^{\infty}\left(\mathbb{R}^{2 N} \backslash \triangle\right)$ and $\nabla \psi \in \mathcal{D}^{\prime}\left(\mathbb{R}^{2 N}\right)$. We have a corresponding maximal domain for the operator $D$ acting on the Hilbert space $L_{\text {sym }}^{2}\left(\mathbb{R}^{2 N}\right)$, which defines the form $T_{\mathrm{A}}$ :

$$
\mathscr{D}_{\mathrm{A}}:=\mathscr{D}\left(D^{\max }\right):=\left\{\psi \in L_{\text {sym }}^{2}: D \psi \in L^{2}\left(\mathbb{R}^{2 N} ; \mathbb{C}^{2 N}\right)\right\}, \quad T_{\mathrm{A}}(\psi)=\frac{1}{2}\|D \psi\|^{2} .
$$

This also defines an associated self-adjoint operator $\hat{T}_{\mathrm{A}}:=\frac{1}{2}\left(D^{\max }\right)^{*} D^{\max }$ with domain

$$
\hat{\mathscr{D}}_{\mathrm{A}}:=\left\{\psi \in \mathscr{D}_{\mathrm{A}}: D \psi \in \mathscr{D}\left(\left(D^{\max }\right)^{*}\right)\right\} .
$$

To emphasize the dependence on $\alpha$, the corresponding domains will be denoted $\hat{\mathscr{D}}_{\mathrm{A}}^{\alpha} \subseteq \mathscr{D}_{\mathrm{A}}^{\alpha}$.

Again, another option is to consider the Friedrichs extension, i.e. define the minimal extension $D^{\text {min }}$ as the closure of $D$ acting on $C_{c}^{\infty}\left(\mathbb{R}^{2 N} \backslash \triangle\right)$,

$$
\mathscr{D}\left(D^{\mathrm{min}}\right):=\overline{C_{c}^{\infty}\left(\mathbb{R}^{2 N} \backslash \triangle\right) \cap L_{\mathrm{sym}}^{2}} \subseteq \mathscr{D}_{\mathrm{A}},
$$

where the closure is taken w.r.t. the graph norm in $L_{\mathrm{sym}}^{2} \times L^{2}$ and the form $\psi \mapsto\|D \psi\|^{2}$. The associated self-adjoint operator is $\hat{T}_{\mathrm{A}}^{\min }:=\frac{1}{2}\left(D^{\mathrm{min}}\right)^{*} D^{\mathrm{min}}$. Note first that for $\alpha=0$, i.e. for the free kinetic energy for bosons, these two definitions coincide, $\hat{T}_{\mathrm{A}}=\hat{T}_{\mathrm{A}}^{\min }=\hat{T}_{0}$, as a consequence of the following lemma.

Lemma 3. We have equality for the Sobolev spaces $H^{1}\left(\mathbb{R}^{2 N}\right)=H_{0}^{1}\left(\mathbb{R}^{2 N} \backslash \triangle\right)$, $H_{\text {sym }}^{1}\left(\mathbb{R}^{2 N}\right)=H_{0, \text { sym }}^{1}\left(\mathbb{R}^{2 N} \backslash \triangle\right), H_{\text {asym }}^{1}\left(\mathbb{R}^{2 N}\right)=H_{0, \text { asym }}^{1}\left(\mathbb{R}^{2 N} \backslash \triangle\right)$, as well as $H^{1}\left(\mathbb{R}^{2 N}\right)=H^{1}\left(\mathbb{R}^{2 N} \backslash \triangle\right)$.

Proof. We first show that $H_{\text {(sym/asym) }}^{1}\left(\mathbb{R}^{2 N}\right)=H_{0, \text { (sym/asym) }}^{1}\left(\mathbb{R}^{2 N} \backslash \triangle\right)$. Since $C_{c}^{\infty}\left(\mathbb{R}^{2 N}\right) \cap L_{\text {(sym/asym) }}^{2}$ is dense in $H^{1}\left(\mathbb{R}^{2 N}\right) \cap L_{\text {(sym/asym) }}^{2}$ we can assume 
that $\psi \in C_{c}^{\infty}\left(\mathbb{R}^{2 N}\right)$. Define $\psi_{\varepsilon}(\mathrm{x}):=\prod_{j<k} \varphi_{\varepsilon}\left(\boldsymbol{x}_{j}-\boldsymbol{x}_{k}\right) \psi(\mathrm{x})$ for $\varepsilon>0$, where $\varphi_{\varepsilon}(\boldsymbol{x}):=\varphi(\varepsilon \ln |\boldsymbol{x}|)$, and $\varphi \in C^{\infty}(\mathbb{R} ;[0,1])$ is taken to be identically zero on $(-\infty,-2)$ and one on $(-1, \infty)$. Then $\psi_{\varepsilon} \in C_{c}^{\infty}\left(\mathbb{R}^{2 N} \backslash \triangle\right) \cap L_{\text {(sym/asym) }}^{2}$,

$$
\left\|\psi_{\varepsilon}-\psi\right\|^{2}=\int_{\mathbb{R}^{2 N}}\left|\prod \varphi_{\varepsilon}-1\right|^{2}|\psi|^{2} d \mathrm{x} \rightarrow 0, \varepsilon \rightarrow 0,
$$

since the measure of $\operatorname{supp}\left(\prod \varphi_{\varepsilon}-1\right) \cap \operatorname{supp} \psi$ tends to zero, and

$$
\begin{aligned}
&\left\|\nabla\left(\psi_{\varepsilon}-\psi\right)\right\|=\|\left(\Pi \varphi_{\varepsilon}-1\right) \nabla \psi+\left(\nabla \Pi \varphi_{\varepsilon}\right) \psi \| \\
& \leq\left\|\left(\Pi \varphi_{\varepsilon}-1\right) \nabla \psi\right\|_{L^{2}(\operatorname{supp} \psi)}+\left\|\nabla \Pi \varphi_{\varepsilon}\right\|_{L^{2}(\operatorname{supp} \psi)}\|\psi\|_{L^{\infty}} .
\end{aligned}
$$

The first term on the r.h.s. tends to zero as above, while for the second we have for each $j \in\{1, \ldots, N\}$

$$
\left\|\nabla_{j} \prod_{k<l} \varphi_{\varepsilon}\left(\boldsymbol{x}_{k}-\boldsymbol{x}_{l}\right)\right\|_{L^{2}(\operatorname{supp} \psi)} \leq \sum_{k \neq j}\left\|\nabla_{j} \varphi_{\varepsilon}\left(\boldsymbol{x}_{j}-\boldsymbol{x}_{k}\right)\right\|_{L^{2}(\operatorname{supp} \psi)}
$$

and

$$
\left\|\nabla_{j} \varphi_{\varepsilon}\left(\boldsymbol{x}_{j}-\boldsymbol{x}_{k}\right)\right\|_{L^{2}(\operatorname{supp} \psi)}^{2} \leq \int_{\operatorname{supp} \psi} \int_{\mathbb{R}^{2}}\left|\nabla \varphi_{\varepsilon}(\boldsymbol{x})\right|^{2} d \boldsymbol{x} \prod_{l \neq j} d \boldsymbol{x}_{l} .
$$

Finally note that, by the substitution $r=e^{s}$,

$$
\frac{1}{2 \pi} \int_{\mathbb{R}^{2}}\left|\nabla \varphi_{\varepsilon}\right|^{2} d \boldsymbol{x}=\varepsilon^{2} \int_{0}^{\infty}\left|\varphi^{\prime}(\varepsilon \ln r)\right|^{2} \frac{d r}{r}=\varepsilon^{2} \int_{-2 / \varepsilon}^{-1 / \varepsilon}\left|\varphi^{\prime}(\varepsilon s)\right|^{2} d s \leq C \varepsilon,
$$

which proves the first part of the lemma.

It remains to show that we can identify $H^{1}\left(\mathbb{R}^{2 N}\right)=H^{1}\left(\mathbb{R}^{2 N} \backslash \triangle\right)$. Any element of the l.h.s. can obviously also be interpreted uniquely as an element of the r.h.s. Conversely, assume that $\psi \in L^{2}\left(\mathbb{R}^{2 N}\right)$ is s.t. the locally defined distribution $\nabla \psi \in \mathcal{D}^{\prime}\left(\mathbb{R}^{2 N} \backslash \triangle\right)$ is actually a function on $\mathbb{R}^{2 N} \backslash \triangle$ which extends to be square-integrable on $\mathbb{R}^{2 N}$. Denote this function by $\mu \in L^{2}\left(\mathbb{R}^{2 N}\right)$. To see that this function is identical to the canonically defined distribution $\nabla \psi \in H^{-1}\left(\mathbb{R}^{2 N}\right)$ when both are considered as distributions on the full space $\mathbb{R}^{2 N}$, let $f:=\nabla \psi-\mu \in H^{-1}\left(\mathbb{R}^{2 N}\right)$ and observe using the first part of the lemma that for any $u \in H^{1}\left(\mathbb{R}^{2 N}\right)=H_{0}^{1}\left(\mathbb{R}^{2 N} \backslash \triangle\right)$, the dual space of $H^{-1}\left(\mathbb{R}^{2 N}\right)$, with $u \leftarrow u_{n} \in C_{c}^{\infty}\left(\mathbb{R}^{2 N} \backslash \triangle\right)$,

$$
\langle f, u\rangle=\lim _{n \rightarrow \infty}\left\langle f, u_{n}\right\rangle=0,
$$

since the distribution $f$ is supported on $\triangle$. Hence $f=0$ in $\mathcal{D}^{\prime}\left(\mathbb{R}^{2 N}\right)$.

Since for $\alpha=0$, by definition $\mathscr{D}\left(D^{\text {max }}\right)=H_{\text {sym }}^{1}\left(\mathbb{R}^{2 N} \backslash \triangle\right)$ and $\mathscr{D}\left(D^{\text {min }}\right)$ $=H_{0, \text { sym }}^{1}\left(\mathbb{R}^{2 N} \backslash \triangle\right)$, we have by the above lemma that $D^{\min }=D^{\max }$ and 
hence obtain the natural kinetic energy operator $\hat{T}_{0}=-\frac{1}{2} \Delta$ for free bosons with domain $\hat{\mathscr{D}}_{\mathrm{A}}^{\alpha=0}=H_{\text {sym }}^{2}\left(\mathbb{R}^{2 N}\right)$. Now, define the unitary multiplication operator $U: L^{2}\left(\mathbb{R}^{2 N}\right) \rightarrow L^{2}\left(\mathbb{R}^{2 N}\right)$,

$$
(U \psi)(\mathrm{x}):=\prod_{j<k} \frac{z_{j}-z_{k}}{\left|z_{j}-z_{k}\right|} \psi(\mathrm{x})=\prod_{j<k} e^{i \phi_{j k}} \psi(\mathrm{x}), \quad \mathrm{x} \in \mathbb{R}^{2 N} \backslash \triangle,
$$

where $\phi_{j k}:=\arg \left(z_{j}-z_{k}\right), z_{j}:=x_{j, 1}+i x_{j, 2}$. We then find that $U: L_{\text {sym }}^{2} \rightarrow$ $L_{\text {asym }}^{2}, L_{\text {asym }}^{2} \rightarrow L_{\text {sym }}^{2}$ is an isomorphism and furthermore, noting that $U$ is smooth on $\mathbb{R}^{2 N} \backslash \triangle$, we have $D^{(\alpha=n+\beta)}=U^{-n} D^{(\alpha=\beta)} U^{n}$ as distributionvalued operators for $n \in \mathbb{Z}$ and $\beta \in \mathbb{R}$. In particular,

$$
D^{(\alpha=2 n)}=U^{-2 n}(-i \nabla) U^{2 n} \quad \text { and } \quad \hat{T}_{\mathrm{A}}^{(\alpha=2 n)}=U^{-2 n} \hat{T}_{0} U^{2 n},
$$

with $\left\|D^{(\alpha=2 n)} \psi\right\|=\left\|\nabla\left(U^{2 n} \psi\right)\right\|$, and therefore

$$
\mathscr{D}_{\mathrm{A}}^{\alpha=2 n}=U^{-2 n} H_{\mathrm{sym}}^{1}\left(\mathbb{R}^{2 N}\right) \quad \text { and } \quad \hat{\mathscr{D}}_{\mathrm{A}}^{\alpha=2 n}=U^{-2 n} H_{\mathrm{sym}}^{2}\left(\mathbb{R}^{2 N}\right) .
$$

Similarly,

$$
\mathscr{D}_{\mathrm{A}}^{\alpha=2 n+1}=U^{-(2 n+1)} H_{\mathrm{asym}}^{1}\left(\mathbb{R}^{2 N}\right) \quad \text { and } \quad \hat{\mathscr{D}}_{\mathrm{A}}^{\alpha=2 n+1}=U^{-(2 n+1)} H_{\text {asym }}^{2}\left(\mathbb{R}^{2 N}\right) .
$$

This also shows that we might have $\nabla \psi \notin L^{2}\left(\mathbb{R}^{2 N}\right)$ although $D \psi \in L^{2}\left(\mathbb{R}^{2 N}\right)$.

The case of general $\alpha \in \mathbb{R}$ is a little trickier. Since $\boldsymbol{A}_{j} \notin L_{\mathrm{loc}}^{2}\left(\mathbb{R}^{2 N}\right)$ we cannot directly apply the standard theorems for magnetic forms (see e.g. Theorems 7.21 and 7.22 in [35, [15] for a related discussion, or [11] for a non-magnetic approach), but they can nevertheless be extended to the anyonic case as follows.

Lemma 4 (Diamagnetic inequality). Assume $\psi \in L^{2}\left(\mathbb{R}^{2 N}\right)$ and $D \psi \in L^{2}\left(\mathbb{R}^{2 N} ; \mathbb{C}^{2 N}\right)$ for arbitrary fixed $\alpha \in \mathbb{R}$. Then $|\psi| \in H^{1}\left(\mathbb{R}^{2 N}\right)$,

$$
\left|\nabla_{j}\right| \psi|(\mathrm{x})| \leq\left|\left(D_{j} \psi\right)(\mathrm{x})\right|
$$

for all $j \in\{1, \ldots, N\}$ and a.e. $\mathrm{x} \in \mathbb{R}^{2 N}$, and

$$
\|\nabla|\psi|\|_{L^{2}\left(\mathbb{R}^{2 N} ; \mathbb{C}^{2 N}\right)} \leq\|D \psi\|_{L^{2}\left(\mathbb{R}^{2 N} ; \mathbb{C}^{2 N}\right)} .
$$

Proof. Note that $|\psi| \in L^{2}\left(\mathbb{R}^{2 N}\right)$ trivially. By the assumptions we have that $D \psi$, considered as a distribution acting on $C_{c}^{\infty}\left(\mathbb{R}^{2 N} \backslash \triangle\right)$, is actually a function locally on $\mathbb{R}^{2 N} \backslash \triangle$ and which is square-integrable on all of $\mathbb{R}^{2 N}$. In particular, $D \psi=-i \nabla \psi+A \psi \in L_{\text {loc }}^{2}\left(\mathbb{R}^{2 N} \backslash \triangle\right)$, where also $A \psi \in L_{\text {loc }}^{2}\left(\mathbb{R}^{2 N} \backslash \triangle\right)$, and hence $-i \nabla \psi \in L_{\text {loc }}^{2}\left(\mathbb{R}^{2 N} \backslash \triangle\right)$, i.e. $\psi \in H_{\text {loc }}^{1}\left(\mathbb{R}^{2 N} \backslash \triangle\right)$. By Theorem 6.17 in [35] we then have $|\psi| \in H_{\text {loc }}^{1}\left(\mathbb{R}^{2 N} \backslash \triangle\right)$ and, for a.e. $\mathrm{x} \in \mathbb{R}^{2 N} \backslash \triangle$,

$$
(\nabla|\psi|)(\mathrm{x})= \begin{cases}\operatorname{Re}\left(\frac{\bar{\psi}}{|\psi|} \nabla \psi\right)(\mathrm{x}), & \text { if } \psi(\mathrm{x}) \neq 0 \\ 0, & \text { if } \psi(\mathrm{x})=0 .\end{cases}
$$


Now, for $j \in\{1, \ldots, N\}$ and each such point $\mathrm{x}$ s.t. $\psi(\mathrm{x}) \neq 0$ we have

$$
\operatorname{Re}\left(\frac{\bar{\psi}}{|\psi|} \nabla_{j} \psi\right)=\operatorname{Re}\left(\frac{\bar{\psi}}{|\psi|} \nabla_{j} \psi+i \boldsymbol{A}_{j} \frac{|\psi|^{2}}{|\psi|}\right)=\operatorname{Re}\left(i \frac{\bar{\psi}}{|\psi|}\left(-i \nabla_{j} \psi+\boldsymbol{A}_{j} \psi\right)\right)
$$

so

$$
\left|\nabla_{j}\right| \psi|| \leq\left|i \frac{\bar{\psi}}{|\psi|}\left(-i \nabla_{j} \psi+\boldsymbol{A}_{j} \psi\right)\right|=\left|-i \nabla_{j} \psi+\boldsymbol{A}_{j} \psi\right|,
$$

while for $\psi(\mathrm{x})=0,(\nabla|\psi|)(\mathrm{x})=0 \leq\left|\left(-i \nabla_{j} \psi+\boldsymbol{A}_{j} \psi\right)(\mathrm{x})\right|$. Hence, (29) holds for a.e. $\mathrm{x} \in \mathbb{R}^{2 N} \backslash \Delta$ (i.e. a.e. $\mathrm{x} \in \mathbb{R}^{2 N}$ ), and

$$
\int_{\mathbb{R}^{2 N}}\left|\nabla_{j}\right| \psi|(\mathrm{x})|^{2} d \mathrm{x} \leq \int_{\mathbb{R}^{2 N}}\left|\left(-i \nabla_{j} \psi+\boldsymbol{A}_{j} \psi\right)(\mathrm{x})\right|^{2} d \mathrm{x} .
$$

We have shown that $|\psi| \in H_{\text {loc }}^{1}\left(\mathbb{R}^{2 N} \backslash \triangle\right)$ with $\int_{\mathbb{R}^{2 N}}|\nabla| \psi||^{2} d \mathrm{x}<\infty$, and hence $|\psi| \in H^{1}\left(\mathbb{R}^{2 N} \backslash \triangle\right)=H^{1}\left(\mathbb{R}^{2 N}\right)$ by Lemma 3 ,

Theorem 5. For arbitrary $\alpha \in \mathbb{R}$ we have that $D^{\min }=D^{\max }$ and hence $\hat{T}_{\mathrm{A}}=\frac{1}{2}\left(D^{\max }\right)^{*} D^{\max }$ is equal to the Friedrichs extension $\hat{T}_{\mathrm{A}}=\frac{1}{2}\left(D^{\mathrm{min}}\right)^{*} D^{\mathrm{min}}$.

Proof. Take $\psi \in \mathscr{D}_{\mathrm{A}}^{\alpha}$. We need to show that $\psi$ can be approximated by a sequence $\psi_{n} \in C_{c}^{\infty}\left(\mathbb{R}^{2 N} \backslash \triangle\right) \cap L_{\text {sym }}^{2}$ s.t. $\left\|\psi_{n}-\psi\right\| \rightarrow 0$ and $\left\|D\left(\psi_{n}-\psi\right)\right\| \rightarrow 0$ as $n \rightarrow \infty$.

Step 1: We start by proving that we without loss of generality can assume $\psi \in L_{c, \text { sym }}^{2}\left(\mathbb{R}^{2 N}\right)$, by finding a sequence $\psi_{n} \in L_{c, \text { sym }}^{2}\left(\mathbb{R}^{2 N}\right)$ s.t. $D \psi_{n} \in$ $L^{2}\left(\mathbb{R}^{2 N}\right),\left\|\psi_{n}-\psi\right\| \rightarrow 0$ and $\left\|D\left(\psi_{n}-\psi\right)\right\| \rightarrow 0$. We take $\psi_{n}:=\chi_{n} \psi$ where $\chi_{n}(\mathrm{x}):=\chi(\mathrm{x} / n)$ and $\chi \in C_{c}^{\infty}\left(\mathbb{R}^{2 N} ;[0,1]\right) \cap L_{\text {sym }}^{2}$ with $\chi \equiv 1$ for $|\mathrm{x}|<1$ and 0 for $|\mathrm{x}|>2$. Then $\psi_{n}$ is symmetric with $\operatorname{supp} \psi_{n} \subseteq B_{2 n}(0)$,

$$
\left\|\psi_{n}-\psi\right\|^{2} \leq \int_{B_{n}(0)^{c}}|\psi|^{2} d \mathrm{x} \rightarrow 0, \quad n \rightarrow \infty,
$$

as well as $D \psi_{n}=-\frac{i}{n} \nabla \chi(\mathrm{x} / n) \psi+\chi_{n} D \psi \in L^{2}\left(\mathbb{R}^{2 N}\right)$, and

$$
\left\|D \psi_{n}-D \psi\right\| \leq \frac{1}{n}\|\nabla \chi(\mathrm{x} / n) \psi\|+\left\|\left(\chi_{n}-1\right) D \psi\right\| \leq \frac{C}{n}\|\psi\|+\|D \psi\|_{L^{2}\left(B_{n}^{c}\right)} \rightarrow 0 .
$$

Step 2: We next show that it is sufficient to assume $\psi \in L_{c, \text { sym }}^{\infty}\left(\mathbb{R}^{2 N}\right)$. By step 1 , we have $\psi \in L_{c, \text { sym }}^{2}\left(\mathbb{R}^{2 N}\right)$ with $D \psi \in L_{c}^{2}\left(\mathbb{R}^{2 N}\right)$. Let $\psi_{n}(\mathrm{x}):=$ $\chi_{n}(|\psi|(\mathrm{x})) \psi(\mathrm{x})$ where $\chi_{n}$ is the cut-off function defined analogously as above but on $\mathbb{R}$. Then $\left|\psi_{n}(\mathrm{x})\right| \leq 2 n$ and $\psi_{n} \in L_{c}^{\infty}\left(\mathbb{R}^{2 N}\right)$. Furthermore, on $\mathbb{R}^{2 N} \backslash \triangle$ we have $\psi \in H_{\text {loc }}^{1}\left(\mathbb{R}^{2 N} \backslash \triangle\right.$ ) and, by Theorem 6.16 in [35] (cp. also Theorem 7.22 in [35]), $\psi_{n} \in H_{\text {loc }}^{1}\left(\mathbb{R}^{2 N} \backslash \triangle\right)$ and

$$
\nabla \psi_{n}=\chi_{n}(|\psi|) \nabla \psi+\psi \chi_{n}^{\prime}(|\psi|) \nabla|\psi| \in L_{\mathrm{loc}}^{2}\left(\mathbb{R}^{2 N} \backslash \triangle\right) .
$$


Hence,

$$
D \psi_{n}=\underbrace{\chi_{n}(|\psi|)}_{\in L^{\infty}} D \psi-i \underbrace{\psi \chi_{n}^{\prime}(|\psi|)}_{\in L^{\infty}} \nabla|\psi| \in L_{\mathrm{loc}}^{2}\left(\mathbb{R}^{2 N} \backslash \triangle\right),
$$

with the full $L^{2}$-norm of $D \psi_{n}$ on $\mathbb{R}^{2 N}$ then bounded by

$$
\left\|\chi_{n}(|\psi|)\right\|_{\infty}\|D \psi\|_{2}+\left\|\psi \chi_{n}^{\prime}(|\psi|)\right\|_{\infty}\|\nabla|\psi|\|_{2} \leq(1+2 n)\|D \psi\|_{2}<\infty,
$$

where we have used the diamagnetic inequality, Lemma 4. Hence, $\psi_{n} \in \mathscr{D}_{\mathrm{A}}^{\alpha}$ and furthermore

$$
\left\|\psi_{n}-\psi\right\|^{2} \leq \int_{|\psi|>n}|\psi|^{2} d \mathrm{x} \rightarrow 0
$$

and

$$
\left\|D \psi_{n}-D \psi\right\| \leq\left\|\left(\chi_{n}(|\psi|)-1\right) D \psi\right\|+\left\|\psi \chi_{n}^{\prime}(|\psi|) \nabla \psi\right\|,
$$

where the first term tends to zero by dominated convergence and for the second we have $|\psi| \chi_{n}^{\prime}(|\psi|) \leq C \chi_{\{|\psi|>n\}}$, hence also $\int_{\mathbb{R}^{2 N}}\left|\psi \chi_{n}^{\prime}(|\psi|)\right|^{2}|\nabla| \psi||^{2} d x \rightarrow$ 0 .

Step 3: Our next step is to show that we can assume $\psi \in L_{c, \text { sym }}^{\infty}\left(\mathbb{R}^{2 N} \backslash \triangle\right)$, i.e. $\psi$ supported away from diagonals. By steps 1-2 we have $\psi \in L_{c, \text { sym }}^{\infty}\left(\mathbb{R}^{2 N}\right)$ and hence $D \psi \in L_{c}^{2}\left(\mathbb{R}^{2 N}\right)$. As in the proof of Lemma 3 we define $\psi_{\varepsilon}(\mathrm{x}):=$ $\prod_{j<k} \varphi_{\varepsilon}\left(\boldsymbol{x}_{j}-\boldsymbol{x}_{k}\right) \psi(\mathrm{x})$ which is in $L_{c, \mathrm{sym}}^{\infty}\left(\mathbb{R}^{2 N} \backslash \triangle\right)$ for $\varepsilon>0$. We obtain

$$
D \psi_{\varepsilon}=\prod \varphi_{\varepsilon} D \psi-i\left(\nabla \prod \varphi_{\varepsilon}\right) \psi \in L_{c}^{2}\left(\mathbb{R}^{2 N} \backslash \triangle\right),
$$

and

$$
\left\|D \psi_{n}-D \psi\right\| \leq\left\|\left(\Pi \varphi_{\varepsilon}-1\right) D \psi\right\|+\left\|\left(\nabla \Pi \varphi_{\varepsilon}\right) \psi\right\|,
$$

where both terms tend to zero as $\varepsilon \rightarrow 0$ as in Lemma 3 .

Step 4: By steps 1-3 we can assume $\psi \in L_{c, \text { sym }}^{\infty}\left(\mathbb{R}^{2 N} \backslash \triangle\right)$. Hence $\psi$ is compactly supported on some open set $\Omega:=\left(\mathbb{R}^{2 N} \backslash \triangle\right) \cap B_{R}(0)$, and also $D \psi=-i \nabla \psi+A \psi \in L_{c}^{2}(\Omega)$. We have then since $A \psi \in L_{c}^{2}(\Omega)$ that $\psi \in H_{0}^{1}(\Omega)$, so there is a sequence $\psi_{n} \in C_{c}^{\infty}(\Omega)$ s.t.

$$
\left\|\psi_{n}-\psi\right\|_{L^{2}(\Omega)} \rightarrow 0, \quad \text { and } \quad\left\|\nabla \psi_{n}-\nabla \psi\right\|_{L^{2}(\Omega)} \rightarrow 0,
$$

as $n \rightarrow \infty$. Hence $D \psi_{n} \in L^{2}\left(\mathbb{R}^{2 N}\right)$ and

$$
\begin{aligned}
\left\|D\left(\psi_{n}-\psi\right)\right\|_{L^{2}\left(\mathbb{R}^{2 N}\right)} & =\left\|-i \nabla\left(\psi_{n}-\psi\right)+A\left(\psi_{n}-\psi\right)\right\|_{L^{2}(\Omega)} \\
\leq & \left\|\nabla \psi_{n}-\nabla \psi\right\|_{L^{2}(\Omega)}+\|A\|_{L^{\infty}(\Omega)}\left\|\psi_{n}-\psi\right\|_{L^{2}(\Omega)} \rightarrow 0
\end{aligned}
$$

which proves the theorem. 


\section{Local exclusion}

Since our wave functions are modeled using bosonic symmetry, but with the non-trivial exchange statistics represented by an interaction, we cannot take advantage of the usual exclusion principle encoded in the total (global) antisymmetry of the wave function. Instead we recall the following local consequence of the Pauli principle for fermions, given as Lemma 5 in [13], and which was used by Dyson and Lenard in their proof of stability of matter in the bulk (see also [14, 32]).

Lemma 6 (Local exclusion for fermions in 3D). Let $\psi \in \bigwedge^{n} L^{2}\left(\mathbb{R}^{3}\right)$ be a wave function of $n$ fermions in $\mathbb{R}^{3}$ and let $\Omega$ be a ball of radius $\ell$. Then

$$
\int_{\Omega^{n}} \sum_{j=1}^{n}\left|\nabla_{j} \psi\right|^{2} d \mathrm{x} \geq(n-1) \frac{\xi^{2}}{\ell^{2}} \int_{\Omega^{n}}|\psi|^{2} d \mathrm{x},
$$

where $\xi \approx 2.082$ is the smallest positive root of the equation

$$
\frac{d^{2}}{d x^{2}} \frac{\sin x}{x}=0 .
$$

We refer to such local energy bounds as a local exclusion principle since it implies that the energy is strictly positive whenever we have more than one particle, and hence that the particles cannot occupy the same singleparticle state (which on a local region with free boundary conditions would be the zero-energy ground state). The inequality follows by expanding $\psi$ in the eigenfunctions of the Neumann Laplacian on $\Omega$, and it turns out to be sufficient with such a weak linear dependence on $n$. A corresponding family of bounds of this form for generalized exchange statistics forms the starting point for our proofs of kinetic energy and Lieb-Thirring inequalities for such statistics.

\subsection{Local exclusion for anyons and bounds for the energy of the ideal anyon gas}

The following local exclusion principle for anyons was proved in [42, starting from a local pairwise relative magnetic Hardy inequality. The method then used for lifting such a pairwise energy bound to a bound for the full kinetic energy on a local region will be illustrated below for the case of intermediate statistics in 1D (and is also in the case of fermions in three dimensions applicable for an alternative proof of Lemma 6 above, up to the value of the constant).

Lemma 7 (Local exclusion for anyons). Let $\psi \in \mathscr{D}_{\mathrm{A}}^{\alpha}$ be a wave function of $n$ anyons and let $\Omega \subseteq \mathbb{R}^{2}$ be either a disk or a square, with area $|\Omega|$. Then

$$
\int_{\Omega^{n}} \sum_{j=1}^{n}\left|D_{j} \psi\right|^{2} d \mathrm{x} \geq(n-1) \frac{c_{\Omega} C_{\alpha, n}^{2}}{|\Omega|} \int_{\Omega^{n}}|\psi|^{2} d \mathrm{x},
$$


where $c_{\Omega}$ is a constant which satisfies $c_{\Omega} \geq 0.169$ for the disk and $c_{\Omega} \geq 0.112$ for the square, and $C_{\alpha, n}$ is defined in (6). Hence, defining the local kinetic energy on $\Omega$ for a normalized $N$-particle wave function $\psi \in \mathscr{D}_{\mathrm{A}}^{\alpha}$

$$
T_{\mathrm{A}}^{\Omega}:=\sum_{j=1}^{N} \int_{\mathbb{R}^{2 N}} \frac{1}{2}\left|D_{j} \psi\right|^{2} \chi_{\Omega}\left(\boldsymbol{x}_{j}\right) d \mathrm{x},
$$

we obtain the following local energy bound in terms of $\rho$

$$
T_{\mathrm{A}}^{\Omega} \geq \frac{c_{\Omega} C_{\alpha, N}^{2}}{2|\Omega|}\left(\int_{\Omega} \rho(\boldsymbol{x}) d \boldsymbol{x}-1\right)_{+} .
$$

We shall consider an immediate application, partially along the lines of [13], of this local exclusion principle for anyons in the form (33) to an explicit lower bound for the ground state energy of a gas of non-interacting anyons. The numerical constant we obtain by this comparatively simple method is much better than the one following from the Lieb-Thirring inequality (58) for anyons given below and proven in [42].

Theorem 8 (Ground state energy for $N$ anyons in a box). Let $\psi \in \mathscr{D}_{\mathrm{A}}^{\alpha}$ be a normalized $N$-anyon wave function supported on a square $Q_{L} \subseteq \mathbb{R}^{2}$ of side length L. Then

$$
T_{\mathrm{A}}=\frac{1}{2} \int_{\mathbb{R}^{2 N}} \sum_{j=1}^{N}\left|D_{j} \psi\right|^{2} d \mathrm{x} \geq \frac{c_{\Omega}}{2} c_{N} C_{\alpha, N}^{2} \frac{N^{2}}{L^{2}},
$$

where $c_{\Omega}$ is is the constant in Lemma 7 for the disk, and

$$
c_{N}:=\sup _{\gamma>0}\left(\frac{1}{\pi \gamma^{2}}-\frac{(1+2 \gamma / \sqrt{N})^{2}}{\pi^{2} \gamma^{4}}\right) .
$$

Hence, the energy per unit area of the ideal anyon gas is bounded below by

$$
\frac{T_{\mathrm{A}}}{L^{2}} \geq \frac{c_{\Omega}}{2} c_{N} C_{\alpha, N}^{2} \bar{\rho}^{2} \stackrel{N>10^{6}}{\geq} 0.021 C_{\alpha, N}^{2} \bar{\rho}^{2},
$$

which, whenever the statistics parameter $\alpha=\frac{\mu}{\nu}$ is an odd numerator reduced fraction, remains finite in the thermodynamic limit and results in the bound

$$
\frac{T_{\mathrm{A}}}{L^{2}} \geq 0.021 \frac{\bar{\rho}^{2}}{\nu^{2}}
$$

as $N \rightarrow \infty$ and $L \rightarrow \infty$ while the total density $\bar{\rho}:=N / L^{2}$ is kept fixed. 
Proof. Our approach is very similar to the proof of Theorem 8 in [13], which used the local exclusion principle (31) for fermions. Introduce an arbitrary length $\ell>0$ and write $Q_{L, \ell}:=Q_{L}+B_{\ell}(0)$. Then,

$$
2 T_{\mathrm{A}}=\int_{\mathbb{R}^{2 N}} \sum_{j=1}^{N}\left|D_{j} \psi\right|^{2} \frac{1}{\pi \ell^{2}} \int_{Q_{L, \ell}} \chi_{B_{\ell}(\boldsymbol{y})}\left(\boldsymbol{x}_{j}\right) d \boldsymbol{y} d \mathrm{x},
$$

and after changing the order of integration and inserting the partition of unity

$$
\begin{aligned}
1=\prod_{k=1}^{N}\left(\chi_{B_{\ell}(\boldsymbol{y})}\left(\boldsymbol{x}_{k}\right)+\chi_{B_{\ell}(\boldsymbol{y})^{c}}\left(\boldsymbol{x}_{k}\right)\right) & \\
= & \sum_{A \subseteq\{1, \ldots, N\}} \prod_{k \in A} \chi_{B_{\ell}(\boldsymbol{y})}\left(\boldsymbol{x}_{k}\right) \prod_{k \notin A} \chi_{B_{\ell}(\boldsymbol{y})^{c}}\left(\boldsymbol{x}_{k}\right),
\end{aligned}
$$

we find

$$
\begin{aligned}
& 2 T_{\mathrm{A}}=\frac{1}{\pi \ell^{2}} \int_{Q_{L, \ell}} \sum_{A \subseteq\{1, \ldots, N\}} \int_{\mathbb{R}^{2 N}} \sum_{j=1}^{N}\left|D_{j} \psi\right|^{2} \chi_{B_{\ell}(\boldsymbol{y})}\left(\boldsymbol{x}_{j}\right) \\
& \times \prod_{k \in A} \chi_{B_{\ell}(\boldsymbol{y})}\left(\boldsymbol{x}_{k}\right) \prod_{k \notin A} \chi_{B_{\ell}(\boldsymbol{y})^{c}}\left(\boldsymbol{x}_{k}\right) d \mathrm{x} d \boldsymbol{y} \\
&=\frac{1}{\pi \ell^{2}} \int_{Q_{L, \ell}} \sum_{A \subseteq\{1, \ldots, N\}} \int_{\left(B_{\ell}(\boldsymbol{y})^{c}\right)^{N-|A|}} \int_{\left(B_{\ell}(\boldsymbol{y})\right)^{|A|}} \sum_{j \in A}\left|D_{j} \psi\right|^{2} \prod_{k \in A} d \boldsymbol{x}_{k} \prod_{k \notin A} d \boldsymbol{x}_{k} d \boldsymbol{y} .
\end{aligned}
$$

We now apply (33) to each term in the first summation above, which involves a partition $A$ of the $N$ particles into $n:=|A|$ of them being inside the disk $B_{\ell}(\boldsymbol{y})$, while the remaining $N-n$ residing outside, and therefore whose contributions to the magnetic potentials $\boldsymbol{A}_{j \in A}$ can be gauged away. Thus, we find

$$
\begin{aligned}
& 2 T_{\mathrm{A}} \geq \frac{c_{\Omega}}{\pi^{2} \ell^{4}} \int_{Q_{L, \ell}} \sum_{A \subseteq\{1, \ldots, N\}} C_{\alpha,|A|}^{2}(|A|-1)_{+} \\
& \times \int_{\left(B_{\ell}(\boldsymbol{y})^{c}\right)^{N-|A|}} \int_{\left(B_{\ell}(\boldsymbol{y})\right)^{|A|}}|\psi|^{2} \prod_{k \in A} d \boldsymbol{x}_{k} \prod_{k \notin A} d \boldsymbol{x}_{k} d \boldsymbol{y} \\
& \geq \frac{c_{\Omega} C_{\alpha, N}^{2}}{\pi^{2} \ell^{4}} \int_{Q_{L, \ell}} \sum_{A \subseteq\{1, \ldots, N\}}|A| \int_{\mathbb{R}^{2 N}}|\psi|^{2} \prod_{k \in A} \chi_{B_{\ell}(\boldsymbol{y})}\left(\boldsymbol{x}_{k}\right) \prod_{k \notin A} \chi_{B_{\ell}(\boldsymbol{y})^{c}}\left(\boldsymbol{x}_{k}\right) d \mathrm{x} d \boldsymbol{y} \\
& -\frac{c_{\Omega} C_{\alpha, N}^{2}}{\pi^{2} \ell^{4}} \int_{Q_{L, \ell}} \int_{\mathbb{R}^{2 N}}|\psi|^{2} \sum_{A \subseteq\{1, \ldots, N\}} \prod_{k \in A} \chi_{B_{\ell}(\boldsymbol{y})}\left(\boldsymbol{x}_{k}\right) \prod_{k \notin A} \chi_{B_{\ell}(\boldsymbol{y})^{c}}\left(\boldsymbol{x}_{k}\right) d \mathrm{x} d \boldsymbol{y}
\end{aligned}
$$


We then revert the above procedure using (38) and

$$
\begin{aligned}
\sum_{A \subseteq\{1, \ldots, N\}} \underbrace{}_{=\sum_{j \in A}^{|A|}} \prod_{k \in A} \chi_{B_{\ell}(\boldsymbol{y})}\left(\boldsymbol{x}_{k}\right) \prod_{k \notin A} \chi_{B_{\ell}(\boldsymbol{y})^{c}}\left(\boldsymbol{x}_{k}\right) \\
=\sum_{j=1}^{N} \sum_{A \subseteq\{1, \ldots, N\}} \chi_{B_{\ell}(\boldsymbol{y})}\left(\boldsymbol{x}_{j}\right) \prod_{k \in A} \chi_{B_{\ell}(\boldsymbol{y})}\left(\boldsymbol{x}_{k}\right) \prod_{k \notin A} \chi_{B_{\ell}(\boldsymbol{y})^{c}}\left(\boldsymbol{x}_{k}\right) \\
=\sum_{j=1}^{N} \chi_{B_{\ell}(\boldsymbol{y})}\left(\boldsymbol{x}_{j}\right),
\end{aligned}
$$

and hence

$$
\begin{aligned}
2 T_{\mathrm{A}} \geq \frac{c_{\Omega} C_{\alpha, N}^{2}}{\pi^{2} \ell^{4}} \int_{\mathbb{R}^{2 N}} \mid & \left.\psi\right|^{2} \int_{Q_{L, \ell}}\left(\sum_{j=1}^{N} \chi_{B_{\ell}(\boldsymbol{y})}\left(\boldsymbol{x}_{j}\right)-1\right) d \boldsymbol{y} d \mathrm{x} \\
& \geq \frac{c_{\Omega} C_{\alpha, N}^{2}}{\pi^{2} \ell^{4}}\left(N \pi \ell^{2}-(L+2 \ell)^{2}\right)=c_{\Omega} C_{\alpha, N}^{2} g(\gamma) \frac{N^{2}}{L^{2}},
\end{aligned}
$$

setting $\ell:=\frac{\gamma L}{\sqrt{N}}$ with $\gamma>0$, and

$$
g(\gamma):=\frac{1}{\pi \gamma^{2}}-\frac{(1+2 \gamma / \sqrt{N})^{2}}{\pi^{2} \gamma^{4}}
$$

This function has a positive maximum $c_{N} \geq 0.0023$ for $N \geq 2$, while $c_{N} \rightarrow$ $1 / 4$ as $N \rightarrow \infty$. In the case $N=1$ we are left with the trivial bound on the energy.

Note that for small $N$ it might be preferable to use the Dirichlet bound for bosons instead (from the diamagnetic inequality (29)):

$$
\sum_{j} \int_{Q_{L}^{N}}\left|D_{j} \psi\right|^{2} d \mathrm{x} \geq \sum_{j} \int_{Q_{L}^{N}}\left|\nabla_{j}\right| \psi||^{2} d \mathrm{x} \geq 2 N \frac{\pi^{2}}{L^{2}} \int_{Q_{L}^{N}}|\psi|^{2} d \mathrm{x} .
$$

This resulting rough - but nevertheless non-trivial - bound (37) for the ideal anyon gas should perhaps be compared with the 'correct' ground state energy per unit volume for a non-interacting gas of density $\bar{\rho}$ of spinless fermions in two dimensions, which in our conventions is

$$
\lim _{N, L \rightarrow \infty} \frac{T_{0}}{L^{2}}=\pi \bar{\rho}^{2} .
$$

Suggestions for improving the constant $c_{\Omega}$ were given in [42]. Also note for comparison that the bound (33) for the disk holds with $c_{\Omega}=\pi \xi^{\prime 2} \approx 10.65$ in the case that $\alpha=1$ (cp. with Lemma 6), where $\xi^{\prime}$ denotes the first zero of the derivative of the Bessel function $J_{1}$. 

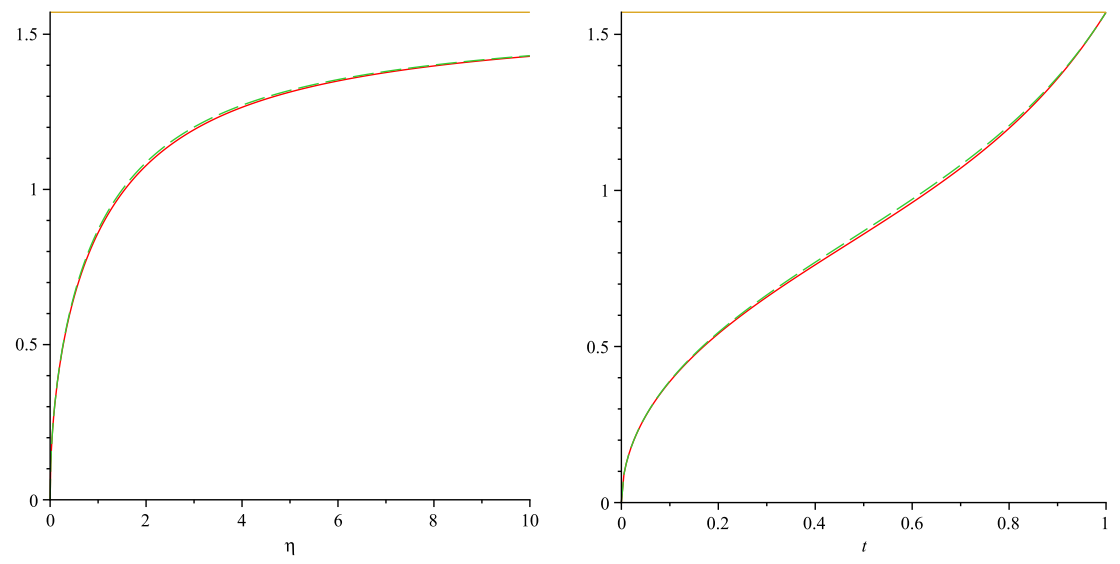

Figure 1: Plot of $\xi_{\mathrm{LL}}(\eta)$ (red solid) and $\arctan \sqrt{\eta+4 \eta^{2} / \pi^{2}}$ (green dashed) as a function of $\eta$ resp. $t=\frac{2}{\pi} \arctan (\eta)$.

\subsection{Local exclusion in one dimension}

As for the anyons [42] we consider the local increase in energy due to the statistical interaction, starting from a pairwise relative consideration.

Lemma 9 (Pairwise relative LL). For $\eta \geq 0$, consider the operator:

$$
H_{\eta}=-\frac{d^{2}}{d r^{2}}+2 \eta \delta_{0}(r)
$$

on a symmetric interval $[-l, l]$ with Neumann boundary conditions at $r= \pm l$. Then $H_{\eta} \geq \xi_{\mathrm{LL}}(\eta l)^{2} / l^{2}$, where $\xi_{\mathrm{LL}}(y)$ is defined as the smallest nonnegative solution to $\xi \tan \xi=y$ given $y \geq 0$.

Proof. Note that for $\eta \geq 0$ there are only non-negative eigenvalues $\lambda$ of $H_{\eta}$. The general solution to the corresponding boundary value problem

$$
\begin{aligned}
-u^{\prime \prime} & =\lambda u, \quad \text { on }(-l, 0) \cup(0, l), \\
u^{\prime}\left(0^{+}\right)=-u^{\prime}\left(0^{-}\right) & =\eta u(0), \\
u^{\prime}(l)=u^{\prime}(-l) & =0,
\end{aligned}
$$

with $u(r)=u(-r)$ and $\lambda \geq 0$, is $u(r)=C\left(\cos \sqrt{\lambda} r+\frac{\eta}{\sqrt{\lambda}} \operatorname{sgn} r \sin \sqrt{\lambda} r\right)$. The condition at $r= \pm l$ demands $\sqrt{\lambda} \tan \sqrt{\lambda} l=\eta$, i.e. $\lambda=\xi(\eta l)^{2} / l^{2}$ is the smallest eigenvalue.

Note that $\xi_{\mathrm{LL}}$ is monotone and $\xi_{\mathrm{LL}}(y) \sim \sqrt{y}$ for small $y$, while $\xi_{\mathrm{LL}}(y) \rightarrow$ $\frac{\pi}{2}$ as $y \rightarrow \infty$. A numerically very good approximation to $\xi_{\mathrm{LL}}$ for all $y$ is (see Figure 1)

$$
\xi_{\mathrm{LL}}(y) \approx \arctan \sqrt{y+\frac{4}{\pi^{2}} y^{2}} .
$$

We shall also find use for the following additional observations: 
Lemma 10. The map $y \mapsto \xi_{\mathrm{LL}}(y)^{2}, y \geq 0$, is monotone and concave, so that for $\eta \geq 0$

$$
\begin{array}{ll}
\xi_{\mathrm{LL}}(\eta / x)^{2} x \geq \xi_{\mathrm{LL}}(\eta)^{2}, & x \geq 1, \\
\xi_{\mathrm{LL}}(\eta / x)^{2} x \leq \xi_{\mathrm{LL}}(\eta)^{2}, & 0 \leq x \leq 1 .
\end{array}
$$

Furthermore, the map $x \mapsto \xi_{\mathrm{LL}}(\eta / x)^{2} x^{3}, x \geq 0$, is monotone and convex for any $\eta \geq 0$.

Proof. Differentiating the identity $\xi \tan \xi=y$ w.r.t. $y>0$ we obtain $\xi^{\prime}\left(\xi^{2}+\right.$ $y(y+1))=\xi$, hence $\xi^{\prime}(y)>0$. Differentiating once more gives

$$
\xi^{\prime \prime}\left(\xi^{2}+y(y+1)\right)=-2 \xi^{\prime}\left(\xi \xi^{\prime}+y\right),
$$

so

$$
\left(\xi^{2}\right)^{\prime \prime}=2 \xi^{\prime 2}+2 \xi \xi^{\prime \prime}=-\frac{4 \xi^{2}}{\xi^{2}+y(y+1)}\left(\frac{\xi^{2}}{\xi^{2}+y(y+1)}+y-\frac{1}{2}\right)<0,
$$

since the expression in brackets is positive for $y>0$. The estimates (41) then follow since $\xi(0)=0$. The proof of convexity for $\xi(\eta / x)^{2} x^{3}$ follows by similar analysis.

Lemma 11 (Pairwise relative CS). For $\alpha \geq 0$, consider nonnegative realizations of the operator:

$$
H_{\alpha}=-\frac{d^{2}}{d r^{2}}+\frac{\alpha(\alpha-1)}{r^{2}}
$$

on a symmetric interval $[-l, l]$ with Neumann boundary conditions at $r=$ $\pm l$. (In the case $-1 / 2<\alpha<3 / 2$ one has to pick the correct self-adjoint extension, matching the b.c. (16) - see below, and Section 2.1.) Then $H_{\alpha} \geq$ $\xi_{\mathrm{CS}}(\alpha)^{2} / l^{2}$, where $\xi_{\mathrm{CS}}(\alpha)$ is defined as the smallest nonnegative solution to

$$
J(\xi)+2 \xi J^{\prime}(\xi)=0,
$$

where $J=J_{\alpha-\frac{1}{2}}$ is the Bessel function of order $\alpha-\frac{1}{2}$.

Proof. The general solution to the corresponding boundary value problem on $[0, l]$ :

$$
\begin{aligned}
-u^{\prime \prime}+\frac{\alpha(\alpha-1)}{r^{2}} u & =\lambda u, \quad \text { on }(0, l), \\
u^{\prime}(l) & =0,
\end{aligned}
$$

is via the ansatz $u:=r^{\frac{1}{2}} v$ found to be (we have assumed $\lambda \geq 0$ and focus on the non-degenerate case $\alpha \neq 1 / 2$ )

$$
u(r)=C_{1} r^{\frac{1}{2}} J_{\alpha-\frac{1}{2}}(\sqrt{\lambda} r)+C_{2} r^{\frac{1}{2}} J_{\frac{1}{2}-\alpha}(\sqrt{\lambda} r) .
$$




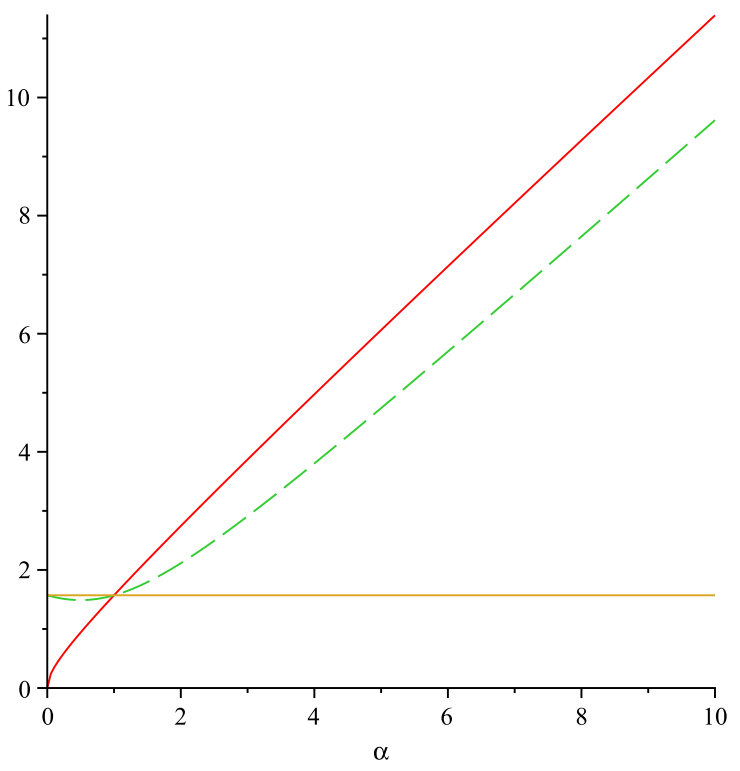

Figure 2: Plot of $\xi_{\mathrm{CS}}(\alpha)$ (red solid) and $\sqrt{\pi^{2} / 4+\alpha(\alpha-1)}$ (green dashed) as a function of $\alpha \geq 0$.

For $\alpha \geq 3 / 2, C_{2}=0$ is enforced by the requirement of square-integrability, while for $\alpha<3 / 2$ this choice is taken to define the domain of $H_{\alpha}$. Note that for $\alpha>1 / 2$ the choice $C_{2}=0$ is required in order for $r^{\alpha}\left(r^{-\alpha} u\right)^{\prime} \in L^{2}([0, l])$ and hence for (a corresponding two-particle version of) $u$ to be in the domain of $Q_{\alpha}^{\max }$. The Neumann condition at $r=l$ then demands $J_{\alpha-\frac{1}{2}}(\sqrt{\lambda} l)+$ $2 \sqrt{\lambda} l J_{\alpha-\frac{1}{2}}^{\prime}(\sqrt{\lambda} l)=0$, i.e. $\lambda=\xi(\alpha)^{2} / l^{2}$ is the smallest eigenvalue.

Note that according to the above $\xi_{\mathrm{CS}}(0)=0$, while $\xi_{\mathrm{CS}}(1)=\frac{\pi}{2}$. A numerically very good approximation to $\xi_{\mathrm{CS}}$ on the interval $[0,1]$ is

$$
\xi_{\mathrm{CS}}(\alpha) \approx \sqrt{\alpha+\left(\frac{\pi^{2}}{4}-1\right) \alpha^{2}}, \quad 0 \leq \alpha \leq 1 .
$$

However, note that $\xi_{\mathrm{CS}}$ continues to grow approximately like $\xi_{\mathrm{CS}}(\alpha) \sim \alpha$ for $\alpha>1$ (see Figure 2). In any case we have from the lowest eigenvalue of the Laplacian with Dirichlet boundary condition at $r=0$, Neumann at $r=l$, and with a potential $\geq \alpha(\alpha-1) / l^{2}$ :

$$
\xi_{\mathrm{CS}}(\alpha) \geq \sqrt{\frac{\pi^{2}}{4}+\alpha(\alpha-1)}, \quad \text { for } \alpha \geq 1 .
$$

Now, consider the Neumann kinetic energy for $n \geq 2$ particles on an 
interval $Q=[a, b]$. Using the identity

$$
n \sum_{j=1}^{n}\left|\partial_{j} \psi\right|^{2}=\sum_{1 \leq j<k \leq n}\left|\partial_{j} \psi-\partial_{k} \psi\right|^{2}+\left|\sum_{j=1}^{n} \partial_{j} \psi\right|^{2}
$$

to separate out the center-of-mass motion, we find

$$
\begin{aligned}
& \int_{Q^{n}} T_{\mathrm{LL} / \mathrm{CS}}^{n}(\psi ; \mathrm{x}) d \mathrm{x} \geq \int_{Q^{n}}\left(\frac{1}{2 n} \sum_{j<k}\left|\partial_{j} \psi-\partial_{k} \psi\right|^{2}+\sum_{j<k} V_{\mathrm{LL} / \mathrm{CS}}\left(x_{j}-x_{k}\right)|\psi|^{2}\right) d \mathrm{x} \\
& \geq \frac{2}{n} \sum_{j<k} \int_{Q^{n-2}} \int_{Q} \int_{[-\delta(R), \delta(R)]}\left(\left|\partial_{r} \psi\right|^{2}+V_{\mathrm{LL} / \mathrm{CS}}(r)|\psi|^{2}\right) d r d R d \mathrm{x}^{\prime} \\
& \geq \frac{2}{n} \sum_{j<k} \int_{Q^{n-2}} \int_{Q} \frac{\xi_{\mathrm{LL} / \mathrm{CS}}^{2}}{\delta(R)^{2}} \int_{[-\delta(R), \delta(R)]}|\psi|^{2} d r d R d \mathrm{x}^{\prime}, \quad \text { (45) }
\end{aligned}
$$

where we for each pair $(j, k)$ split the parameterization of $Q^{n}$ into $n-2$ variables $\mathrm{x}^{\prime}=\left(x_{1}, \ldots, \not_{j}, \ldots, \not x_{k}, \ldots, x_{N}\right)$, a pairwise center-of-mass coordinate $R:=\left(x_{j}+x_{k}\right) / 2 \in Q$, and a relative coordinate $r:=\left(x_{j}-x_{k}\right) \in$ $[-\delta(R), \delta(R)]$. We have set $\delta(R):=2 \min \{|R-a|,|R-b|\}$, and in the above we also used that $V_{\mathrm{LL} / \mathrm{CS}} \geq 0$ followed by Lemma 9 or 11 .

In the Calogero-Sutherland case $\xi_{\mathrm{CS}}=\xi_{\mathrm{CS}}(\alpha)$ is constant on the interval $Q$ and a local exclusion principle follows in a similar way as for the anyons:

Lemma 12 (Local exclusion for CS). We have for $\alpha \geq 1$

$$
\int_{Q^{n}} T_{\mathrm{CS}}^{n}(\psi ; \mathrm{x}) d \mathrm{x} \geq(n-1) \frac{\xi_{\mathrm{CS}}(\alpha)^{2}}{|Q|^{2}} \int_{Q^{n}}|\psi|^{2} d \mathrm{x},
$$

and hence, defining the local kinetic energy on $Q$ for a normalized $N$-particle wave function $\psi \in \mathscr{D}_{\mathrm{CS}}^{\alpha}$

$$
T_{\mathrm{CS}}^{Q}:=\sum_{j=1}^{N} \int_{\mathbb{R}^{N}} \frac{1}{2}\left(\left|\partial_{j} \psi\right|^{2}+\sum_{(j \neq) k=1}^{N} V_{\mathrm{CS}}\left(x_{j}-x_{k}\right)|\psi|^{2}\right) \chi_{Q}\left(x_{j}\right) d \mathrm{x},
$$

we obtain the local energy bound

$$
T_{\mathrm{CS}}^{Q} \geq \frac{\xi_{\mathrm{CS}}(\alpha)^{2}}{|Q|^{2}}\left(\int_{Q} \rho(x) d x-1\right)_{+} .
$$

Proof. We use $\delta(R)^{-2} \geq|Q|^{-2}$ in (45) to obtain (46). Then we proceed as for Lemma 8 in [42] (cp. also [13] and the proof of Theorem 8 above), inserting the partition of unity

$$
1=\sum_{A \subseteq\{1, \ldots, N\}} \prod_{l \in A} \chi_{Q}\left(x_{l}\right) \prod_{l \notin A} \chi_{Q^{c}}\left(x_{l}\right)
$$


in (47):

$$
\begin{gathered}
\sum_{A} \int_{\mathbb{R}^{N}} \frac{1}{2} \sum_{j \in A}\left(\left|\partial_{j} \psi\right|^{2}+\sum_{(j \neq) k=1}^{N} V_{\mathrm{CS}}\left(x_{j}-x_{k}\right)|\psi|^{2}\right) \prod_{l \in A} \chi_{Q}\left(x_{l}\right) \prod_{l \notin A} \chi_{Q^{c}}\left(x_{l}\right) d \mathrm{x} \\
\geq \sum_{A} \int_{\left(Q^{c}\right)^{N-|A|}} \int_{Q^{|A|}} \frac{1}{2}\left(\sum_{j \in A}\left|\partial_{j} \psi\right|^{2}+\sum_{j \neq k \in A} V_{\mathrm{CS}}\left(x_{j}-x_{k}\right)|\psi|^{2}\right) \prod_{l \in A} d x_{l} \prod_{l \notin A} d x_{l} \\
\geq \sum_{A}(|A|-1) \frac{\xi_{\mathrm{CS}}(\alpha)^{2}}{|Q|^{2}} \int_{\left(Q^{c}\right)^{N-|A|}} \int_{Q^{|A|}}|\psi|^{2} \prod_{l \in A} d x_{l} \prod_{l \notin A} d x_{l} \\
=\frac{\xi_{\mathrm{CS}}(\alpha)^{2}}{|Q|^{2}} \int_{\mathbb{R}^{N}}\left(\sum_{j=1}^{N} \chi_{Q}\left(x_{j}\right)-1\right)|\psi|^{2} d \mathrm{x}
\end{gathered}
$$

hence (48), where we again used $V_{\mathrm{CS}} \geq 0$ and in the last step the partition of unity (49).

In the Lieb-Liniger case, we observe from Lemma 10 with $x:=|Q| / \delta(R) \geq 1$ that

$$
\frac{\xi_{\mathrm{LL}}(\eta \delta(R))^{2}}{\delta(R)^{2}} \geq \frac{\xi_{\mathrm{LL}}(\eta|Q| / x)^{2} x}{|Q|^{2}} \geq \frac{\xi_{\mathrm{LL}}(\eta|Q|)^{2}}{|Q|^{2}} \quad \text { for all } R \in Q,
$$

and can thereafter proceed exactly as above for the Calogero-Sutherland case.

Lemma 13 (Local exclusion for LL). We have for $\eta \geq 0$

$$
\int_{Q^{n}} T_{\mathrm{LL}}^{n}(\psi ; \mathrm{x}) d \mathrm{x} \geq(n-1) \frac{\xi_{\mathrm{LL}}(\eta|Q|)^{2}}{|Q|^{2}} \int_{Q^{n}}|\psi|^{2} d \mathrm{x},
$$

and hence, defining the local kinetic energy on $Q$ for a normalized $N$-particle wave function $\psi \in \mathscr{D}_{\mathrm{LL}}^{\eta}$

$$
T_{\mathrm{LL}}^{Q}:=\sum_{j=1}^{N} \int_{\mathbb{R}^{N}} \frac{1}{2}\left(\left|\partial_{j} \psi\right|^{2}+\sum_{(j \neq) k=1}^{N} V_{\mathrm{LL}}\left(x_{j}-x_{k}\right)|\psi|^{2}\right) \chi_{Q}\left(x_{j}\right) d \mathrm{x},
$$

we obtain the local energy bound

$$
T_{\mathrm{LL}}^{Q} \geq \frac{\xi_{\mathrm{LL}}(\eta|Q|)^{2}}{|Q|^{2}}\left(\int_{Q} \rho(x) d x-1\right)_{+} .
$$

Proof. Use the estimate (50) in (45) and continue as in the proof of Lemma 12. 


\section{Local uncertainty}

We shall use the following local form of the uncertainty principle on a $d$ dimensional cube $Q$.

Lemma 14 (Local uncertainty principle). For any $\varepsilon \in(0,1)$ we have

$$
T^{Q} \geq \frac{C_{d}^{\prime}}{2} \varepsilon^{1+4 / d} \frac{\int_{Q} \rho^{1+2 / d}}{\left(\int_{Q} \rho\right)^{2 / d}}-\frac{C_{d}^{\prime}}{2}\left(1+\left(\frac{\varepsilon}{1-\varepsilon}\right)^{1+4 / d}\right) \frac{\int_{Q} \rho}{|Q|^{2 / d}},
$$

where the local kinetic energy $T^{Q}$ denotes either $T_{\mathrm{LL}}^{Q}$ with $\eta \geq 0$, or $T_{\mathrm{CS}}^{Q}$ with $\alpha \geq 1$, or $T_{\mathrm{A}}^{Q}$ with any $\alpha \in \mathbb{R}$, or simply $T_{0}^{Q}$, the free kinetic energy on a $d$-cube $Q$ in any dimension $d \geq 1$ (i.e. half of the l.h.s. of (55)).

This follows from the following lemma concerning the free kinetic energy for an arbitrary $N$-particle wave function, which was proved as Theorem 14 in [42] using methods from [52].

Lemma 15 (Uncertainty on a cube). Let $Q$ be a cube in $\mathbb{R}^{d}$ with volume $|Q|$, and let $u \in H^{1}\left(\mathbb{R}^{d N}\right)$ be an arbitrary $N$-particle wave function. Then

$$
\sum_{j=1}^{N} \int_{\mathbb{R}^{d N}}\left|\nabla_{j} u\right|^{2} \chi_{Q}\left(\boldsymbol{x}_{j}\right) d \mathrm{x} \geq \frac{C_{d}^{\prime}}{\left(\int_{Q} \rho\right)^{2 / d}} \int_{Q}\left[\rho(\boldsymbol{x})^{\frac{1}{2}}-\left(\frac{\int_{Q} \rho}{|Q|}\right)^{\frac{1}{2}}\right]_{+}^{\frac{2(d+2)}{d}} d \boldsymbol{x}
$$

where $\rho(\boldsymbol{x}):=\sum_{j=1}^{N} \int_{\mathbb{R}^{d(N-1)}}\left|u\left(\boldsymbol{x}_{1}, \ldots, \boldsymbol{x}_{j-1}, \boldsymbol{x}, \boldsymbol{x}_{j+1}, \ldots, \boldsymbol{x}_{N}\right)\right|^{2} \prod_{k \neq j} d \boldsymbol{x}_{k}$.

The constant $C_{d}^{\prime}:=d^{2} C_{d}^{-\frac{2}{d}} /(d+2)(d+4)$, with $C_{d}$ given below, enters via a bound for the growth of the sequence of eigenvalues $\left\{\lambda_{k}\right\}_{k=0}^{\infty}$ for the Neumann Laplacian on $Q$ :

$$
\sum_{0<\lambda_{k}<e} \frac{2^{d}}{|Q|} \leq C_{d} e^{\frac{d}{2}}, \quad e \geq 0 .
$$

In the case $d=1$ we have $\lambda_{k}=\frac{\pi^{2}}{|Q|^{2}} k^{2}, k=0,1,2, \ldots$, and

$$
\#\left\{k: 0<\lambda_{k}<e\right\}=\#\left\{k \in \mathbb{Z}: 0<k<e^{\frac{1}{2}}|Q| / \pi\right\} \leq e^{\frac{1}{2}}|Q| / \pi,
$$

and hence we take $C_{1}:=2 / \pi$ and $C_{1}^{\prime}=\pi^{2} / 60$. In the case $d \geq 2$ we have $\lambda_{\boldsymbol{k}}=\pi^{2}|\boldsymbol{k}|^{2} /|Q|^{\frac{2}{d}}, \boldsymbol{k} \in \mathbb{Z}_{\geq 0}^{d}$, and by geometric considerations

$$
\#\left\{\boldsymbol{k}: 0<\lambda_{\boldsymbol{k}}<e\right\}=\#\left\{\boldsymbol{k} \in \mathbb{Z}_{\geq 0}^{d}: 0<|\boldsymbol{k}|<e^{\frac{1}{2}}|Q|^{\frac{1}{d}} / \pi\right\} \leq d\left(e^{\frac{1}{2}}|Q|^{\frac{1}{d}} / \pi\right)^{d},
$$

i.e. the optimal constant in (56) is $C_{d}:=d 2^{d} / \pi^{d}$, so

$$
C_{d}^{\prime}=\frac{\pi^{2}}{4} \frac{d^{2-2 / d}}{(d+2)(d+4)}
$$

In particular, $C_{2}=8 / \pi^{2}$ and $C_{2}^{\prime}=\pi^{2} / 48$. We remark that these constants are probably far from the sharp ones in the inequality (55). 
Proof of Lemma 14. We reduce to the case of free bosons, $T^{Q} \geq T_{0}^{Q}$, by discarding the statistics potentials or using the diamagnetic inequality (Lemma (4) $\left|D_{j} \psi\right| \geq\left|\nabla_{j}\right| \psi||$ for anyons (which also preserves $\rho$ ). Then, from Lemma 15.

$$
T_{0}^{Q} \geq \frac{1}{2} \frac{C_{d}^{\prime}}{\left(\int_{Q} \rho\right)^{2 / d}} \int_{Q}\left[\rho(\boldsymbol{x})^{\frac{1}{2}}-\left(\frac{\int_{Q} \rho}{|Q|}\right)^{\frac{1}{2}}\right]_{+}^{2+4 / d} d \boldsymbol{x}
$$

with

$$
\begin{aligned}
& \int_{Q}\left[\rho(\boldsymbol{x})^{\frac{1}{2}}-\left(\frac{\int_{Q} \rho}{|Q|}\right)^{\frac{1}{2}}\right]_{+}^{2+4 / d} d \boldsymbol{x} \\
& \geq \int_{Q}\left|\rho(\boldsymbol{x})^{\frac{1}{2}}-\left(\frac{\int_{Q} \rho}{|Q|}\right)^{\frac{1}{2}}\right|^{2+4 / d} d \boldsymbol{x}-\int_{Q}\left(\frac{\int_{Q} \rho}{|Q|}\right)^{1+2 / d} d \boldsymbol{x} \\
&=\left\|\rho^{\frac{1}{2}}-\left(\frac{\int_{Q} \rho}{|Q|}\right)^{\frac{1}{2}}\right\|_{2+4 / d}^{2+4 / d}-\frac{\left(\int_{Q} \rho\right)^{1+2 / d}}{|Q|^{2 / d}},
\end{aligned}
$$

where the norm $\|\cdot\|_{p}$ is that of $L^{p}(Q)$. The first term is bounded below by $\left(\left\|\rho^{\frac{1}{2}}\right\|_{2+4 / d}-\left\|\left(\int_{Q} \rho /|Q|\right)^{\frac{1}{2}}\right\|_{2+4 / d}\right)^{2+4 / d}$ using the triangle inequality. Furthermore, by convexity we have for any $a, b \in \mathbb{R}, \varepsilon \in(0,1)$, and $p \geq 1$ that

$$
(\varepsilon a+(1-\varepsilon) b)^{p} \leq \varepsilon a^{p}+(1-\varepsilon) b^{p},
$$

and hence with $a=A-B$ and $b=\frac{\varepsilon}{1-\varepsilon} B$,

$$
(A-B)^{p} \geq \varepsilon^{p-1} A^{p}-\left(\frac{\varepsilon}{1-\varepsilon}\right)^{p-1} B^{p} .
$$

Applying this inequality to the norms above with $p=2+4 / d$, we finally arrive at (54).

We remark that bounds of the form (54) could also be obtained by application of standard Sobolev and Poincaré inequalities, but would then need to be dealt with differently for $d=1,2$ and $d \geq 3$ (see [17] concerning the latter case). For $d=2$ an alternative form of the local uncertainty principle than (54) was given and used in [42].

\section{$5 \quad$ Lieb-Thirring inequalities}

\subsection{A Lieb-Thirring inequality for anyons}

In [42] we introduced an approach to Lieb-Thirring inequalities based on local exclusion and uncertainty, resulting in the following energy bounds for 
anyons in $\mathbb{R}^{2}$.

Theorem 16 (Kinetic energy inequality for anyons). Let $\psi \in \mathscr{D}_{\mathrm{A}}^{\alpha}, \alpha \in \mathbb{R}$, be a normalized $N$-anyon wave function on $\mathbb{R}^{2}$. Then

$$
\int_{\mathbb{R}^{2 N}} T_{\mathrm{A}}^{N}(\psi ; \mathrm{x}) d \mathrm{x} \geq C_{\mathrm{A}} C_{\alpha, N}^{2} \int_{\mathbb{R}^{2}} \rho(\boldsymbol{x})^{2} d \boldsymbol{x},
$$

for some positive constant $10^{-4} \leq C_{\mathrm{A}} \leq \pi$.

Corollary (Lieb-Thirring inequality for anyons). Let $\psi \in \mathscr{D}_{\mathrm{A}}^{\alpha}$ be a normalized $N$-anyon wave function and $V$ a real-valued potential on $\mathbb{R}^{2}$. Then

$$
\int_{\mathbb{R}^{2 N}}\left(T_{\mathrm{A}}^{N}(\psi ; \mathrm{x})+\sum_{j=1}^{N} V\left(\boldsymbol{x}_{j}\right)|\psi|^{2}\right) d \mathrm{x} \geq-C_{\mathrm{A}}^{\prime} C_{\alpha, N}^{-2} \int_{\mathbb{R}^{2}}\left|V_{-}(\boldsymbol{x})\right|^{2} d \boldsymbol{x},
$$

for a positive constant $C_{\mathrm{A}}^{\prime}=\left(4 C_{\mathrm{A}}\right)^{-1}$.

The rough lower bound $C_{\mathrm{A}} \geq 10^{-4}$ that is given here for the optimal constant $C_{\mathrm{A}}$ in (58) can be computed in a similar way as we do below for the Lieb-Liniger case (in the proof of the corresponding Theorem 11 in [42] one can take e.g. $\epsilon=1 / 7$ and $\kappa=C_{\alpha, N}^{2} / 12$ ). Note that from (58) follows also the bound for the free anyon gas (see [42])

$$
\frac{T_{\mathrm{A}}}{|\Omega|} \geq C_{\mathrm{A}} C_{\alpha, N}^{2} \bar{\rho}^{2}
$$

with mean density $\bar{\rho}=N /|\Omega|$, assuming that $\rho$ is supported on a domain $\Omega \subseteq \mathbb{R}^{2}$. Comparing with (39) it is clear that the optimal constant in (58) and (60) satisfies $C_{\mathrm{A}} \leq C_{\mathrm{A}}^{\mathrm{cl}}:=\pi$, which is the exact constant for fermions in the semi-classical approximation. We should also compare with Theorem 8 which produced a significantly better numerical bound for the constant for the anyon gas.

\subsection{Lieb-Thirring inequalities in one dimension}

By proceeding as for the anyons, combining local uncertainty with local exclusion, we can prove the following for intermediate statistics in one dimension, summarized in Theorem 1.

Theorem 17 (Lieb-Thirring inequalities for 1D Lieb-Liniger). For $\psi \in \mathscr{D}_{\mathrm{LL}}^{\eta}$ with $\eta \geq 0$ and $N \geq 1$ we have

$$
\int_{\mathbb{R}^{N}} T_{\mathrm{LL}}^{N}(\psi ; \mathrm{x}) d \mathrm{x} \geq C_{\mathrm{LL}} \int_{\mathbb{R}} \xi_{\mathrm{LL}}\left(2 \eta / \rho^{*}(x)\right)^{2} \rho(x)^{3} d x,
$$


for some positive constant $3 \cdot 10^{-5} \leq C_{\mathrm{LL}} \leq 2 / 3$, where $\rho^{*}$ is the HardyLittlewood maximal function of $\rho$ (see (170) ). In particular, if $\frac{\int_{Q} \rho}{|Q|} \leq \gamma \bar{\rho}$ for all intervals $Q$ and some $\gamma>0$, then

$$
\begin{gathered}
\int_{\mathbb{R}^{N}} T_{\mathrm{LL}}^{N}(\psi ; \mathrm{x}) d \mathrm{x} \geq C_{\mathrm{LL}} \xi_{\mathrm{LL}}(2 \eta /(\gamma \bar{\rho}))^{2} \int_{\mathbb{R}} \rho(x)^{3} d x \\
\int_{\mathbb{R}^{N}}\left(T_{\mathrm{LL}}^{N}(\psi ; \mathrm{x})+\sum_{j=1}^{N} V\left(x_{j}\right)|\psi|^{2}\right) d \mathrm{x} \geq-\frac{C_{\mathrm{LL}}^{\prime}}{\xi_{\mathrm{LL}}(2 \eta /(\gamma \bar{\rho}))} \int_{\mathbb{R}}\left|V_{-}(x)\right|^{\frac{3}{2}} d x
\end{gathered}
$$

with $C_{\mathrm{LL}}^{\prime}:=\frac{2}{3}\left(3 C_{\mathrm{LL}}\right)^{-\frac{1}{2}}$, and if $\rho$ is supported on an interval of length $L$

$$
T_{\mathrm{LL}} / L \geq C_{\mathrm{LL}} \xi_{\mathrm{LL}}(2 \eta /(\gamma \bar{\rho}))^{2} \bar{\rho}^{3}, \quad \bar{\rho}:=N / L .
$$

Proof. For $N=1$ and $N=2$ we use $T_{\mathrm{LL}} \geq T_{0}$ and Lemma 15 (which generalizes to $Q=\mathbb{R}$ ) with a resulting constant $C_{1}^{\prime} / 8 \geq 0.020$.

For $N \geq 3$ we can consider the kinetic energy $T_{\mathrm{LL}}^{Q}$ on an arbitrary finite interval $Q_{0} \subset \mathbb{R}$ s.t. $\int_{Q_{0}} \rho \geq 2$. We split the interval $Q_{0}$ in halves iteratively, organizing the resulting subintervals $Q$ in a (full binary) tree $\mathbb{T}$; cp. [42]. The procedure can be arranged so that $Q_{0}$ is finally covered by intervals $Q_{B}$ marked B s.t. $2 \leq \int_{Q_{B}} \rho<4$, and $Q_{A}$ marked A s.t. $0 \leq \int_{Q_{A}} \rho<2$, sitting at the leaves of the tree and s.t. at least one B-interval is at the highest level of every branch of the tree. On the B-intervals we use local exclusion, (53), together with local uncertainty, Lemma 14, to obtain

$$
T_{\mathrm{LL}}^{Q_{B}} \geq \kappa \frac{C_{1}^{\prime}}{2} \varepsilon^{5} \frac{\int_{Q_{B}} \rho^{3}}{4^{2}}-\kappa \frac{C_{1}^{\prime}}{2}\left(1+\left(\frac{\varepsilon}{1-\varepsilon}\right)^{5}\right) \frac{4}{\left|Q_{B}\right|^{2}}+(1-\kappa) \frac{\xi_{\mathrm{LL}}\left(\eta\left|Q_{B}\right|\right)^{2}}{\left|Q_{B}\right|^{2}}
$$

for any $\varepsilon, \kappa \in(0,1)$. For simplicity we set $\varepsilon=\frac{1}{2}$ and $\kappa=\frac{1}{2} \xi_{\mathrm{LL}}\left(\eta\left|Q_{B}\right|\right)^{2} /(\pi / 2)^{2}$ $\leq \frac{1}{2}$ to find

$$
T_{\mathrm{LL}}^{Q_{B}} \geq \xi_{\mathrm{LL}}\left(\eta\left|Q_{B}\right|\right)^{2}\left(c_{1} \int_{Q_{B}} \rho^{3}+\frac{c_{2}}{\left|Q_{B}\right|^{2}}\right)
$$

with $c_{1}=2 / \pi^{2} \cdot C_{1}^{\prime} / 2^{10}=2^{-11} / 15$ and $c_{2}=-2 / \pi^{2} \cdot 4 C_{1}^{\prime}+1 / 2=11 / 30$.

Note by monotonicity of $\xi_{\mathrm{LL}}$ that

$$
\xi_{\mathrm{LL}}\left(\eta\left|Q_{B}\right|\right) \geq \xi_{\mathrm{LL}}(2 \eta / \tilde{\rho}(x)), \quad \text { where }\left.\quad \tilde{\rho}\right|_{Q_{B}}:=\frac{\int_{Q_{B}} \rho}{\left|Q_{B}\right|}
$$

i.e. $\tilde{\rho}$ is defined to be the mean of $\rho$ on each B-interval. The A-intervals are further divided into a subclass $\mathrm{A}_{2}$ on which

$$
\int_{Q_{A}} \rho^{3}>c \frac{\left(\int_{Q_{A}} \rho\right)^{3}}{\left|Q_{A}\right|^{2}}
$$


with $c:=2^{7}$, so that by Lemma 14 (again using $\varepsilon=\frac{1}{2}$ )

$$
T_{Q_{A_{2}}} \geq c_{3} \int_{Q_{A_{2}}} \rho^{3}=\frac{4 c_{3}}{\pi^{2}} \int_{Q_{A_{2}}} \xi_{\mathrm{LL}}(2 \eta / \tilde{\rho})^{2} \rho^{3}, \quad \text { where }\left.\quad \tilde{\rho}\right|_{Q_{A_{2}}}:=0
$$

$c_{3}=\frac{1}{2} C_{1}^{\prime} / 2^{8}=\pi^{2} c_{1}$, and a remaining subclass $\mathrm{A}_{1}$ for which

$$
\int_{Q_{A}} \rho^{3} \leq c \frac{\left(\int_{Q_{A}} \rho\right)^{3}}{\left|Q_{A}\right|^{2}}
$$

Consider the set $\mathcal{A}_{1}\left(Q_{B}\right)$ of such intervals $Q_{A_{1}}$ which can be found by going back in the tree $\mathbb{T}$ from a fixed B-interval $Q_{B}$ at level $k \in \mathbb{N}$ (possibly all the way to $Q_{0}$ ), and then one step forward. On each level $1 \leq j \leq k$ there is at most one such interval which we denote by $Q_{j}$ (and otherwise we can define $\left|Q_{j}\right|:=\left|Q_{0}\right| / 2^{j}$ for the below expressions to make sense). Using Lemma 10 we have

$$
\xi_{\mathrm{LL}}\left(\eta\left|Q_{B}\right| /\left(\left|Q_{B}\right| /\left|Q_{j}\right|\right)\right)^{2} \frac{\left|Q_{B}\right|}{\left|Q_{j}\right|} \leq \xi_{\mathrm{LL}}\left(\eta\left|Q_{B}\right|\right)^{2}
$$

and hence

$$
\begin{array}{r}
\sum_{j=1}^{k} \xi_{\mathrm{LL}}\left(\eta\left|Q_{j}\right|\right)^{2} \frac{\left|Q_{B}\right|^{2}}{\left|Q_{j}\right|^{2}} \leq \sum_{j=1}^{k} \xi_{\mathrm{LL}}\left(\eta\left|Q_{B}\right|\right)^{2} \frac{\left|Q_{B}\right|}{\left|Q_{j}\right|}=\xi_{\mathrm{LL}}\left(\eta\left|Q_{B}\right|\right)^{2} \sum_{j=1}^{k} \frac{2^{-k}}{2^{-j}} \\
\leq 2 \xi_{\mathrm{LL}}\left(\eta\left|Q_{B}\right|\right)^{2}
\end{array}
$$

Defining $\left.\tilde{\rho}\right|_{Q_{A_{1}}}:=\int_{Q_{A_{1}}} \rho /\left|Q_{A_{1}}\right|$ to be the mean also on $\mathrm{A}_{1}$-intervals, we have by $0 \leq \tilde{\rho} \leq 2 /\left|Q_{A_{1}}\right|$ and monotonicity of $\xi_{\mathrm{LL}}(\eta / x)^{2} x^{3}$ that

$$
\int_{Q_{A_{1}}} \xi_{\mathrm{LL}}(2 \eta / \tilde{\rho})^{2} \rho^{3} \leq \xi_{\mathrm{LL}}(2 \eta / \tilde{\rho})^{2} c \tilde{\rho}^{3}\left|Q_{A_{1}}\right| \leq c \xi_{\mathrm{LL}}\left(\eta\left|Q_{A_{1}}\right|\right)^{2} \frac{2^{3}}{\left|Q_{A_{1}}\right|^{2}},
$$

and therefore by the above

$$
\sum_{Q_{A_{1}} \in \mathcal{A}_{1}\left(Q_{B}\right)} \int_{Q_{A_{1}}} \xi_{\mathrm{LL}}(2 \eta / \tilde{\rho})^{2} \rho^{3} \leq \sum_{j=1}^{k} 8 c \frac{\xi_{\mathrm{LL}}\left(\eta\left|Q_{j}\right|\right)^{2}}{\left|Q_{j}\right|^{2}} \leq 16 c \frac{\xi_{\mathrm{LL}}\left(\eta\left|Q_{B}\right|\right)^{2}}{\left|Q_{B}\right|^{2}}
$$

for every B-interval $Q_{B}$. In other words the energy on all intervals with almost constant low density is dominated by that from exclusion on the B-intervals. Using (66) we then have

$$
T_{\mathrm{LL}}^{Q_{B}} \geq c_{1} \int_{Q_{B}} \xi_{\mathrm{LL}}(2 \eta / \tilde{\rho})^{2} \rho^{3}+c_{2}^{\prime} \sum_{Q_{A_{1}} \in \mathcal{A}_{1}\left(Q_{B}\right)} \int_{Q_{A_{1}}} \xi_{\mathrm{LL}}(2 \eta / \tilde{\rho})^{2} \rho^{3},
$$

$c_{2}^{\prime}=c_{2} /(16 c)=2^{-12} \cdot 11 / 15$, and hence by (67) and (68)

$$
T_{\mathrm{LL}} \geq T_{\mathrm{LL}}^{Q_{0}}=\sum_{Q_{A, B} \in \mathbb{T}} T_{\mathrm{LL}}^{Q_{A, B}} \geq C_{\mathrm{LL}} \int_{Q_{0}} \xi_{\mathrm{LL}}(2 \eta / \tilde{\rho})^{2} \rho^{3},
$$


where $C_{\mathrm{LL}}:=\min \left\{c_{1}, c_{2}^{\prime}, 4 c_{3} / \pi^{2}\right\}=c_{1}>3 \cdot 10^{-5}$. We now use $\tilde{\rho} \leq \rho^{*}$, with

$$
\rho^{*}(x):=\sup \left\{\frac{\int_{Q} \rho}{|Q|}: Q \text { is a finite interval containing } x\right\}
$$

the (uncentered) Hardy-Littlewood maximal function of $\rho$, and finally let $Q_{0}$ approach $\mathbb{R}$ to obtain (61).

The bounds (63) and (64) follow in a standard way from (62) (see e.g. Theorem 4.3 in [37]). The bound $C_{\mathrm{LL}} \leq 2 / 3$ for the optimal constant in (61) follows by taking the limit $\eta \rightarrow+\infty$ and comparing (64) with the semiclassics for fermions.

Theorem 18 (Lieb-Thirring inequalities for 1D Calogero-Sutherland). Let $\psi \in \mathscr{D}_{\mathrm{CS}}^{\alpha}$ with $\alpha \geq 1$ and $N \geq 2$. Given any finite interval $Q_{0}$ s.t. $\int_{Q_{0}} \rho \geq 2$, we have

$$
T_{\mathrm{CS}}^{Q_{0}} \geq C_{\mathrm{CS}} \xi_{\mathrm{CS}}(\alpha)^{2} \int_{Q_{0}} \tilde{\rho}(x)^{3} d x \geq C_{\mathrm{CS}} \xi_{\mathrm{CS}}(\alpha)^{2} \frac{\left(\int_{Q_{0}} \rho(x) d x\right)^{3}}{\left|Q_{0}\right|^{2}},
$$

for some positive constant $1 / 32 \leq C_{\mathrm{CS}} \leq 2 / 3$, and $\tilde{\rho}$ defined below (see (74)) as a piecewise constant approximation to $\rho$. In particular,

$$
T_{\mathrm{CS}}=\int_{\mathbb{R}^{N}} T_{\mathrm{CS}}^{N}(\psi ; \mathrm{x}) d \mathrm{x} \geq C_{\mathrm{CS}} \xi_{\mathrm{CS}}(\alpha)^{2} \int_{\mathbb{R}} \tilde{\rho}(x)^{3} d x,
$$

and if $\rho$ is confined to a length $L$

$$
T_{\mathrm{CS}} / L \geq C_{\mathrm{CS}} \xi_{\mathrm{CS}}(\alpha)^{2} \bar{\rho}^{3}, \quad \bar{\rho}:=N / L .
$$

Here the assumption $N>1$ is important because of the dependence on $\alpha$ in the bound (72). Note that the integral in the r.h.s. of (72) actually becomes a Riemann sum approximation of $\int_{\mathbb{R}} \rho(x)^{3} d x$ in the limit of many particles with relatively uniform density. Also note that the usual LiebThirring inequality (2) for fermions (with a constant independent of $\alpha$ ) is valid as a lower bound for $T_{\mathrm{CS}}$ for all $N$ and $\alpha \geq 1$. However, we will prove below that $\tilde{\rho}$ cannot be replaced with the actual density $\rho$ in the r.h.s. of (72) as long as $C_{\mathrm{CS}}$ is a positive constant independent of $\alpha$, using that $\xi_{\mathrm{CS}}(\alpha)$ can become arbitrarily large with $\alpha \rightarrow \infty$.

Proof. We split an arbitrary finite interval $Q_{0}$ s.t. $\int_{Q_{0}} \rho \geq 2$ iteratively as in the proof of the previous theorem (in the case $N=2$ we can approximate this condition arbitrarily well with a finite interval, and some minor adjustments to the constants below will need to be incorporated accordingly). In the Calogero-Sutherland case we cannot combine (48) with Lemma 14 to yield a uniform bound w.r.t. $\alpha$ (better than the one for fermions) since $\xi_{\mathrm{CS}}(\alpha) \rightarrow \infty$ as $\alpha \rightarrow \infty$. Instead we need to rely solely on energy from exclusion (also 
resulting in a slightly better lower bound for the constant $\left.C_{\mathrm{CS}}\right)$. We define $\left.\tilde{\rho}\right|_{Q_{0}^{c}}:=0$ and

$$
\left.\tilde{\rho}\right|_{Q}:=\frac{\int_{Q} \rho}{|Q|}
$$

for all A- and B-intervals $Q \in \mathbb{T}$, and use for $0<\kappa<1$

$$
T_{\mathrm{CS}}^{Q_{B}} \geq \frac{\xi_{\mathrm{CS}}(\alpha)^{2}}{\left|Q_{B}\right|^{2}}\left(\int_{Q_{B}} \rho-1\right)_{+} \geq \xi_{\mathrm{CS}}(\alpha)^{2}\left(\kappa \frac{3}{4^{3}} \int_{Q_{B}} \tilde{\rho}^{3}+(1-\kappa) \frac{1}{\left|Q_{B}\right|^{2}}\right)
$$

(with $x-1 \geq 3 x^{3} / 4^{3}$ when $2 \leq x:=\int_{Q_{B}} \rho \leq 4$ ) for the B-intervals, and

$$
\sum_{Q_{A} \in \mathcal{A}\left(Q_{B}\right)} \int_{Q_{A}} \tilde{\rho}^{3}=\sum_{j=1}^{k} \frac{\left(\int_{Q_{j}} \rho\right)^{3}}{\left|Q_{j}\right|^{2}} \leq \sum_{j=1}^{k} \frac{8}{4^{-j}\left|Q_{0}\right|^{2}} \leq \frac{8}{3} \frac{4^{k+1}}{\left|Q_{0}\right|^{2}}=\frac{2^{5} / 3}{\left|Q_{B}\right|^{2}}
$$

for all the A-intervals associated to a B-interval $Q_{B}$ at level $k$ in $\mathbb{T}$ (similarly as for $\mathcal{A}_{1}\left(Q_{B}\right)$ in the previous proof). Hence,

$$
T_{\mathrm{CS}}^{Q_{B}} \geq \xi_{\mathrm{CS}}(\alpha)^{2}\left(\kappa \frac{3}{2^{6}} \int_{Q_{B}} \tilde{\rho}^{3}+(1-\kappa) \frac{3}{2^{5}} \sum_{Q_{A} \in \mathcal{A}\left(Q_{B}\right)} \int_{Q_{A}} \tilde{\rho}^{3}\right),
$$

and

$$
T_{\mathrm{CS}} \geq T_{\mathrm{CS}}^{Q_{0}} \geq \sum_{Q_{B} \in \mathbb{T}} T_{\mathrm{CS}}^{Q_{B}} \geq C_{\mathrm{CS}} \xi_{\mathrm{CS}}(\alpha)^{2} \int_{Q_{0}} \tilde{\rho}^{3},
$$

where $\kappa:=2 / 3, C_{\mathrm{CS}}:=2^{-5}$.

For (71) and (73) we use that $\int_{Q_{0}} \tilde{\rho}^{3} \geq\left|Q_{0}\right|^{-2}\left(\int_{Q_{0}} \tilde{\rho}\right)^{3}$ and $\int_{Q_{0}} \tilde{\rho}=\int_{Q_{0}} \rho$. The upper bound on the optimal constant $C_{\mathrm{CS}}$ follows by comparing (73) with $\alpha=1$ to the semiclassical limit for fermions.

Theorem 19 (Necessity of $\tilde{\rho}$ in (72)). Assume that for some nonnegative constant $C_{\mathrm{CS}}$ the inequality

$$
\int_{\mathbb{R}^{N}} T_{\mathrm{CS}}^{N}(\psi ; \mathrm{x}) d \mathrm{x} \geq C_{\mathrm{CS}} \xi_{\mathrm{CS}}(\alpha)^{2} \int_{\mathbb{R}} \rho(x)^{3} d x
$$

holds for all $\psi \in \mathscr{D}_{\mathrm{CS}}^{\alpha}$ and $\alpha \geq 1$. Then we must have $C_{\mathrm{CS}}=0$.

Proof. The idea is to spread out the particles one by one but with high individual localization, and then compare the behavior of the left and right hand sides of (76) as $\alpha \rightarrow \infty$. Let $\varphi \in C_{c}^{\infty}\left([-1,1] ; \mathbb{R}_{\geq 0}\right)$ be a bump function normalized s.t. $\int_{-1}^{1} \varphi^{2} d x=1$, and define $\varphi_{\varepsilon}(x):=\varepsilon^{-\frac{1}{2}} \varphi(x / \varepsilon)$, and the completely symmetric $N$-particle wave function

$$
\psi(\mathrm{x}):=\frac{1}{\sqrt{N !}} \sum_{\sigma \in S_{N}} \prod_{j=1}^{N} \varphi_{\varepsilon}\left(x_{j}-\sigma(j)\right) .
$$


Assuming $0<\varepsilon<1 / 3$ we have on the support of $\psi$ that $\left|x_{j}-x_{k}\right|>1 / 3$ for all $j \neq k$, so this wave function is certainly in the domain of $T_{\mathrm{CS}}$. It is furthermore normalized:

$$
\int_{\mathbb{R}^{N}}|\psi|^{2} d \mathrm{x}=\frac{1}{N !} \sum_{\sigma, \tau \in S_{N}} \int_{\mathbb{R}^{N}} \prod_{j=1}^{N} \underbrace{\varphi_{\varepsilon}\left(x_{j}-\sigma(j)\right) \varphi_{\varepsilon}\left(x_{j}-\tau(j)\right)}_{\delta_{\sigma(j), \tau(j)} \varphi_{\varepsilon}\left(x_{j}-\sigma(j)\right)^{2}} d \mathrm{x}=1,
$$

with one-particle density

$$
\begin{aligned}
& \rho(x)=N \int_{\mathbb{R}^{N-1}}\left|\psi\left(x, x_{2}, \ldots, x_{N}\right)\right|^{2} d \mathrm{x}^{\prime} \\
&=\frac{N}{N !} \sum_{\sigma, \tau \in S_{N}} \int_{\mathbb{R}^{N-1}} \varphi_{\varepsilon}(x-\sigma(1)) \varphi_{\varepsilon}(x-\tau(1)) \prod_{j=2}^{N} \varphi_{\varepsilon}\left(x_{j}-\sigma(j)\right) \varphi_{\varepsilon}\left(x_{j}-\tau(j)\right) d \mathrm{x}^{\prime} \\
&=\sum_{j=1}^{N} \varphi_{\varepsilon}(x-j)^{2},
\end{aligned}
$$

supported on the interval $[0, N+1]$, and s.t. $\rho(x)^{3}=\sum_{j=1}^{N} \varphi_{\varepsilon}(x-j)^{6}$. Furthermore,

$$
\int_{\mathbb{R}^{N}} \sum_{j<k} \frac{\alpha(\alpha-1)}{\left(x_{j}-x_{k}\right)^{2}}|\psi|^{2} d \mathrm{x}<9 \alpha(\alpha-1)\left(\begin{array}{c}
N \\
2
\end{array}\right)
$$

and

$$
\partial_{k} \psi=\frac{\varepsilon^{-1}}{\sqrt{N !}} \sum_{\sigma \in S_{N}} \varepsilon^{-\frac{1}{2}} \varphi^{\prime}\left(\left(x_{j}-\sigma(k)\right) / \varepsilon\right) \prod_{j \neq k} \varphi_{\varepsilon}\left(x_{j}-\sigma(j)\right),
$$

implying $\int_{\mathbb{R}^{N}}\left|\partial_{k} \psi\right|^{2} d \mathrm{x}=\varepsilon^{-2} \int_{-1}^{1}\left(\varphi^{\prime}\right)^{2} d x$. Hence,

$$
T_{\mathrm{CS}}<\varepsilon^{-2} N \int_{-1}^{1}\left(\varphi^{\prime}\right)^{2} d x+\frac{9}{2} \alpha(\alpha-1) N(N-1)
$$

while the r.h.s. of (176) is

$$
C_{\mathrm{CS}} \xi_{\mathrm{CS}}(\alpha)^{2} \sum_{j=1}^{N} \int_{\mathbb{R}} \varepsilon^{-3} \varphi((x-j) / \varepsilon)^{6} d x=\varepsilon^{-2} N C_{\mathrm{CS}} \xi_{\mathrm{CS}}(\alpha)^{2} \int_{-1}^{1} \varphi^{6} d x
$$

Now, taking $\varepsilon$ depending on $N$ so small that $\varepsilon^{-2} N C_{\mathrm{CS}} \int_{-1}^{1} \varphi^{6} d x>9 N(N-$ 1 ), we find using (43), $\xi_{\mathrm{CS}}(\alpha)^{2}>\alpha(\alpha-1)$, that (78) is strictly greater than (77) for $\alpha$ sufficiently large, unless $C_{\mathrm{CS}}=0$.

Note on the other hand that $\tilde{\rho}$, and hence the r.h.s. of (72), is independent of $\varepsilon$ for the above choice of $\psi$. 


\section{Some applications}

\subsection{Many anyons in a harmonic oscillator potential}

As an application of the kinetic energy inequality (58) for anyons, we can consider the ground state energy of $N$ anyons in a harmonic oscillator potential, a problem which has been discussed substantially in the literature. The Hamiltonian operator is in our conventions given by

$$
H=\sum_{j=1}^{N}\left(\frac{1}{2} D_{j} \cdot D_{j}+V\left(\boldsymbol{x}_{j}\right)\right), \quad V(\boldsymbol{x}):=\frac{\omega^{2}}{2}|\boldsymbol{x}|^{2} .
$$

For $N=2$ the exact spectrum was computed already in [29], while for $N \geq 3$ the ground state energy is only known exactly for fermions $\alpha=1$ and in a small neighborhood around bosons $\alpha=0$. The lower part of the spectrum has been computed numerically for the full range of $\alpha \in[0,1]$ for $N=3,4$ [56, 44, 57] and for general $N$ there are some known families of exact but excited or singular eigenstates [62, 10, 5]. Some general features extend to arbitrary $N$, such as large numbers of level crossings in the ground state as $N$ is fixed large and $\alpha$ varied [9]. See [26] for a more recent summary of the status of this problem.

With the kinetic energy inequality (58) at hand, we would like to find a lower bound for the quadratic form

$$
\begin{aligned}
\langle\psi, H \psi\rangle=\int_{\mathbb{R}^{2 N}} \sum_{j}\left(\frac{1}{2}\left|D_{j} \psi\right|^{2}+V\left(\boldsymbol{x}_{j}\right)|\psi|^{2}\right) d \mathrm{x} & \\
& \quad \geq \int_{\mathbb{R}^{2}}\left(C_{\mathrm{A}} C_{\alpha, N}^{2} \rho(\boldsymbol{x})^{2}+\frac{\omega^{2}}{2}|\boldsymbol{x}|^{2} \rho(\boldsymbol{x})\right) d \boldsymbol{x}
\end{aligned}
$$

subject to the conditions $\int_{\mathbb{R}^{2}} \rho=N$ and $\rho \geq 0$. We extremize the functional

$$
F[\rho, \lambda]:=\int_{\mathbb{R}^{d}}\left(C_{\mathrm{A}} C_{\alpha, N}^{2} \rho(\boldsymbol{x})^{2}+\frac{\omega^{2}}{2}|\boldsymbol{x}|^{2} \rho(\boldsymbol{x})-\lambda \rho(\boldsymbol{x})\right) d \boldsymbol{x}+\lambda N
$$

to find

$$
2 C_{\mathrm{A}} C_{\alpha, N}^{2} \rho(\boldsymbol{x})+\frac{\omega^{2}}{2}|\boldsymbol{x}|^{2}-\lambda=0, \quad \text { and } \quad N=\int_{\mathbb{R}^{2}} \rho(\boldsymbol{x}) d \boldsymbol{x},
$$

i.e.

$$
\rho(\boldsymbol{x})=\frac{\left[\lambda-\omega^{2}|\boldsymbol{x}|^{2} / 2\right]_{+}}{2 C_{\mathrm{A}} C_{\alpha, N}^{2}},
$$

with

$2 C_{\mathrm{A}} C_{\alpha, N}^{2} N=2 \pi \int_{0}^{\sqrt{2 \lambda} / \omega}\left(\lambda-\omega^{2} r^{2} / 2\right) r d r=2 \pi\left[\lambda \frac{r^{2}}{2}-\omega^{2} \frac{r^{4}}{8}\right]_{0}^{\sqrt{2 \lambda} / \omega}=\frac{\pi \lambda^{2}}{\omega^{2}}$, 
and hence $\lambda=\omega C_{\alpha, N} \sqrt{2 C_{\mathrm{A}} N / \pi}$. Plugging in this form for $\rho$, the r.h.s. of (179) is

$$
\begin{aligned}
& \frac{2 \pi}{2 C_{\mathrm{A}} C_{\alpha, N}^{2}} \int_{0}^{\sqrt{2 \lambda} / \omega}\left(\frac{1}{2}\left(\lambda-\omega^{2} r^{2} / 2\right)^{2}+\frac{\omega^{2}}{2} r^{2}\left(\lambda-\omega^{2} r^{2} / 2\right)\right) r d r \\
&=\frac{\pi}{C_{\mathrm{A}} C_{\alpha, N}^{2}}\left[\frac{\lambda^{2}}{2} \frac{r^{2}}{2}+\frac{\omega^{4}}{4}\left(\frac{1}{2}-1\right) \frac{r^{6}}{6}\right]_{0}^{\sqrt{2 \lambda} / \omega}=\frac{\pi}{2 C_{\mathrm{A}} C_{\alpha, N}^{2}}\left(\frac{\lambda^{3}}{\omega^{2}}-\frac{\lambda^{3}}{3 \omega^{2}}\right) \\
&=\frac{\pi\left(\omega C_{\alpha, N} \sqrt{2 C_{\mathrm{A}} N} / \sqrt{\pi}\right)^{3}}{3 C_{\mathrm{A}} C_{\alpha, N}^{2} \omega^{2}}
\end{aligned}
$$

and hence

$$
\langle\psi, H \psi\rangle \geq \frac{1}{3} \sqrt{\frac{8 C_{\mathrm{A}}}{\pi}} C_{\alpha, N} \omega N^{\frac{3}{2}} .
$$

This resulting bound for the energy in terms of $C_{\alpha, N} \omega N^{\frac{3}{2}}$ allows us to compare the graph of $\alpha \mapsto C_{\alpha, N}$ (the limiting graph for $C_{\alpha}:=\lim _{N \rightarrow \infty} C_{\alpha, N}$ is sketched in Figure 2 in [42]) with the previously found exact and numerical spectra for 2-4 anyons and the extensions of certain features in such spectra to more anyons (note e.g. the partial reflection symmetry about $\alpha=1 / 2$; cp. [53]). The correct ground state energy in the case $\alpha=1$ is to leading order $\sim \frac{\sqrt{8}}{3} \omega N^{\frac{3}{2}}$. We also note that for bosons, which satisfy the weaker kinetic energy inequality $T_{0} \geq \frac{C_{\mathrm{A}}}{N} \int_{\mathbb{R}^{2}} \rho^{2}$, the above bound reduces to

$$
\langle\psi, H \psi\rangle \geq \frac{1}{3} \sqrt{\frac{8 C_{\mathrm{A}}}{\pi}} \omega N .
$$

The correct ground state energy for bosons is of course $\omega N$.

The bound (80) improves, for odd numerator rational $\alpha$, the previously known best lower bound for the energy of $N$ anyons in a harmonic oscillator [53, 9]:

$$
\langle\psi, H \psi\rangle \geq \omega\left(N+\left|L+\alpha \frac{N(N-1)}{2}\right|\right),
$$

where $L$ is the total angular momentum of the state $\psi ;\left(-i \sum_{j} \boldsymbol{x}_{j} \wedge \nabla_{j}\right) \psi=$ $L \psi$. We see from this inequality that the energy actually grows like $N^{2}$, unless $L \sim-\alpha\left(\begin{array}{c}N \\ 2\end{array}\right)$ (equality is possible for certain $N$ and $\alpha$ ) for which (81) reduces to the bosonic bound for the energy. The non-trivial dependence on $\alpha$ and $N$ of these energy bounds, together with other aspects of the spectra mentioned above, raises the question whether the limiting constant in the kinetic energy inequality (58) actually cannot be improved for even numerator and irrational values of $\alpha$ (for which $C_{\alpha, N} \rightarrow 0$ as $N \rightarrow \infty$ ). We refer to [43] for further discussion on this possibility. 


\subsection{Stability of odd-fractional anyonic matter with Coulomb interactions}

In the context of the fractional quantum Hall effect it is relevant to consider anyons arising in the form of quasiparticles with fractional charge and statistics [2] embedded in a three-dimensional system, possibly with an effective 3D Coulomb repulsion. Since these are then arising in a material there could also be a large number of oppositely charged particles which impose a Coulomb attraction on the anyons. We can ask if such an interacting system is stable in the thermodynamic limit, i.e. if the negative ground state energy grows at most linearly with the total number of particles (cp. stability for ordinary fermionic matter [37]). Consider therefore the model Hamiltonian

$$
\begin{gathered}
H(N, \mathrm{R}):=\frac{1}{2 m} \sum_{j=1}^{N} D_{j} \cdot D_{j}+V_{C}(\mathrm{x}, \mathrm{R}), \\
V_{C}(\mathrm{x}, \mathrm{R}):=\sum_{1 \leq j<k \leq N} \frac{1}{\left|\boldsymbol{x}_{j}-\boldsymbol{x}_{k}\right|}-\sum_{j=1}^{N} \sum_{k=1}^{K} \frac{Z}{\left|\boldsymbol{x}_{j}-\boldsymbol{R}_{k}\right|}+\sum_{1 \leq j<k \leq K} \frac{Z^{2}}{\left|\boldsymbol{R}_{j}-\boldsymbol{R}_{k}\right|},
\end{gathered}
$$

which can be taken to describe $N$ anyons with mass $m$ and unit charge -1 moving in the plane $\mathbb{R}^{2}$, together with $K$ static 'nuclei' of charge $Z \geq 1$ at positions $\mathrm{R}=\left(\boldsymbol{R}_{1}, \ldots, \boldsymbol{R}_{K}\right) \in \mathbb{R}^{2 K}$, and where all particles are interacting through electromagnetic forces in $3 \mathrm{D}$. The condition $Z \geq 1$ is only technical and can be relaxed, while the overall scale of the unit charge can be adjusted with the variable mass $m$.

By the usual electromagnetic screening in 3D, we have the following important lemma to simplify the problem (given e.g. as Theorem 5.4 in [37]).

Lemma 20 (Baxter's electrostatic inequality).

$$
V_{C}(\mathrm{x}, \mathrm{R}) \geq-(2 Z+1) \sum_{j=1}^{N} \frac{1}{d_{R}\left(\boldsymbol{x}_{j}\right)}+\frac{Z^{2}}{8} \sum_{k=1}^{K} \frac{1}{D_{k}},
$$

where $D_{k}:=\frac{1}{2} \min _{j \neq k}\left|\boldsymbol{R}_{j}-\boldsymbol{R}_{k}\right|$, and $d_{\mathrm{R}}(\boldsymbol{x}):=\min _{k=1, \ldots, K}\left|\boldsymbol{x}-\boldsymbol{R}_{k}\right|$.

Hence, we would like to find a lower bound, independent of $\mathrm{R}$, for the quadratic form

$$
\begin{aligned}
& \langle\psi, H(N, \mathrm{R}) \psi\rangle=\int_{\mathbb{R}^{2 N}}\left(\frac{1}{2 m} \sum_{j}\left|D_{j} \psi\right|^{2}+V_{C}(\mathrm{x}, \mathrm{R})|\psi|^{2}\right) d \mathrm{x} \\
& \geq \frac{1}{2 m} \int_{\mathbb{R}^{2 N}} \sum_{j}\left(\left|D_{j} \psi\right|^{2}-2 m \frac{2 Z+1}{d_{\mathrm{R}}\left(\boldsymbol{x}_{j}\right)}|\psi|^{2}\right) d \mathrm{x}+\frac{Z^{2}}{8} \sum_{k=1}^{K} \frac{1}{D_{k}} .
\end{aligned}
$$


With the Lieb-Thirring inequality (59) for anyons at hand, we will prove the following theorem.

Theorem 21 (Stability of 'anyonic matter' for odd-fractional statistics). For a normalized $N$-anyon wave function $\psi \in \mathscr{D}_{\mathrm{A}}^{\alpha}$, with $\alpha=\mu / \nu$ an odd numerator reduced fraction, we have the lower bound

$$
\langle\psi, H(N, \mathrm{R}) \psi\rangle \geq-C \nu^{2} m Z^{2}(K+N),
$$

for some positive constant $C$.

There are some technical difficulties related to the dimensionality. In order to handle the non-square-integrability of the one-particle potential in (82) close to the nuclei, we split the integral into two regions. On a region $B_{\varepsilon}:=\bigcup_{k=1}^{K} B_{\varepsilon_{k}}\left(\boldsymbol{R}_{k}\right), 0<\varepsilon_{k} \leq D_{k}$, close to the nuclei we use $\left|D_{j} \psi\right| \geq$ $\left|\nabla_{j}\right| \psi||$ together with the following lemma to deduce

$$
\begin{aligned}
\int_{\mathbb{R}^{2 N}} & \left(\frac{1}{2}\left|D_{j} \psi\right|^{2}-2 m \frac{2 Z+1}{d_{\mathrm{R}}\left(\boldsymbol{x}_{j}\right)}|\psi|^{2}\right) \chi_{B_{\boldsymbol{\varepsilon}}}\left(\boldsymbol{x}_{j}\right) d \mathrm{x} \\
& \geq-2 m(2 Z+1) \int_{\mathbb{R}^{2 N}}\left(4 m(2 Z+1)+\frac{2}{\varepsilon\left(\boldsymbol{x}_{j}\right)}\right)|\psi|^{2} \chi_{B_{\boldsymbol{\varepsilon}}}\left(\boldsymbol{x}_{j}\right) d \mathrm{x},
\end{aligned}
$$

where $\varepsilon(\boldsymbol{x}):=\varepsilon_{k}$ on the disk $B_{\varepsilon_{k}}\left(\boldsymbol{R}_{k}\right)$ around the $k$ th nucleus.

Lemma 22. For $v \in H^{1}\left(B_{\varepsilon}(0)\right)$ and $\varepsilon, \mu>0$ we have

$$
\int_{B_{\varepsilon}(0)}\left(\mu|\nabla v|^{2}-\frac{1}{|\boldsymbol{r}|}|v|^{2}\right) d \boldsymbol{r} \geq-\left(\frac{1}{\mu}+\frac{2}{\varepsilon}\right) \int_{B_{\varepsilon}(0)}|v|^{2} d \boldsymbol{r} .
$$

Proof. We simply adapt the proof of Lemma 2 in [13] to two dimensions. It is clear that the ratio

$$
\int_{B_{\varepsilon}(0)}\left(\mu|\nabla v|^{2}-\frac{1}{|\boldsymbol{r}|}|v|^{2}\right) d \boldsymbol{r} / \int_{B_{\varepsilon}(0)}|v|^{2} d \boldsymbol{r}
$$

is minimized by a radially symmetric function $v(r)$, and that we may consider a lower bound when the domain is replaced by the annulus $B_{\varepsilon}(0) \backslash B_{\delta}(0)$ if the bound is uniform in $\delta>0$. We have therefore reduced to the Neumann problem

$$
-\mu\left(v^{\prime \prime}+\frac{v^{\prime}}{r}\right)-\frac{v}{r}=\lambda v, \quad v^{\prime}(\delta)=v^{\prime}(\varepsilon)=0,
$$

on $[\delta, \varepsilon]$, and would like to show that the lowest eigenvalue $\lambda \geq-\frac{1}{\mu}-\frac{2}{\varepsilon}$. Noting that the ground state $v$ is nodeless and defining $\omega:=-v^{\prime} / v$, we have

$$
\frac{\mu}{r} \partial_{r}(r \omega)-\mu \omega^{2}-\frac{1}{r}=\lambda, \quad \omega(\delta)=\omega(\varepsilon)=0,
$$


and after multiplying by $r$ and integrating over $[\delta, \varepsilon]$,

$$
0-\mu \int_{\delta}^{\varepsilon} \omega^{2} r d r-(\varepsilon-\delta)=\lambda \frac{\varepsilon^{2}-\delta^{2}}{2} .
$$

Now, differentiating the differential equation in (84) by $r$ we have

$$
\omega^{\prime \prime}+\omega^{\prime}\left(\frac{1}{r}-2 \omega\right)+\frac{1}{r^{2}}\left(\frac{1}{\mu}-\omega\right)=0,
$$

and therefore an extremal point $r_{0} \in(\delta, \varepsilon)$ for $\omega$ cannot be a minimum if $\omega\left(r_{0}\right)<0$ and cannot be a maximum if $\omega\left(r_{0}\right)>1 / \mu$, hence $0 \leq \omega \leq 1 / \mu$. It then follows by (85) that $\lambda \geq-\frac{1}{\mu}-\frac{2}{\varepsilon+\delta}$, which proves the lemma.

Now, combining the integral term in (82) with (83) using half of the kinetic energy, produces the lower bound

$$
\begin{aligned}
& \langle\psi, H(N, \mathrm{R}) \psi\rangle \geq \\
& \frac{1}{2 m} \int_{\mathbb{R}^{2 N}} \sum_{j}\left(\frac{1}{2}\left|D_{j} \psi\right|^{2}-2 m(2 Z+1)\left(2\left(2 m(2 Z+1)+\varepsilon^{-1}\right) \chi_{B_{\varepsilon}}\left(\boldsymbol{x}_{j}\right)\right.\right. \\
& \left.\left.\quad+\frac{\chi_{B_{\varepsilon}} c\left(\boldsymbol{x}_{j}\right)}{d_{\mathrm{R}}\left(\boldsymbol{x}_{j}\right)}\right)|\psi|^{2}\right) d \mathrm{x} \\
& \geq \frac{1}{2 m} \int_{\mathbb{R}^{2 N}} \sum_{j}\left(\frac{1}{2}\left|D_{j} \psi\right|^{2}-V_{1}\left(\boldsymbol{x}_{j}\right)|\psi|^{2}\right) d \mathrm{x}-(2 Z+1) b N
\end{aligned}
$$

with the one-particle potential $V_{1}(\boldsymbol{x}):=$

$$
2 m(2 Z+1)\left(2\left(2 m(2 Z+1)+\frac{1}{\varepsilon(\boldsymbol{x})}\right) \chi_{B_{\boldsymbol{\varepsilon}}}(\boldsymbol{x})+\left(\frac{1}{d_{\mathrm{R}}(\boldsymbol{x})}-b\right) \chi_{B_{\boldsymbol{\varepsilon}}}(\boldsymbol{x})\right),
$$

where we have here also used a standard method to handle the non-squareintegrability of the potential at infinity, by adding and subtracting a constant $b>0$. Applying the Lieb-Thirring inequality (59) with the resulting potential $V_{1}$ given above, we find

$$
\begin{gathered}
\langle\psi, H(N, \mathrm{R}) \psi\rangle \\
\geq-2 m C_{\mathrm{A}}^{\prime} \nu^{2}(2 Z+1)^{2} \int_{\mathbb{R}^{2}}\left[2\left(2 m(2 Z+1)+\varepsilon^{-1}\right) \chi_{B_{\boldsymbol{\varepsilon}}}+\left(\frac{1}{d_{\mathrm{R}}}-b\right) \chi_{B_{\boldsymbol{\varepsilon}}}\right]_{+}^{2} \\
-(2 Z+1) b N+\frac{Z^{2}}{8} \sum_{k=1}^{K} \frac{1}{D_{k}} \\
\geq-2 m C_{\mathrm{A}}^{\prime} \nu^{2}(2 Z+1)^{2} \sum_{k=1}^{K}\left(\int_{B_{\varepsilon_{k}}\left(\boldsymbol{R}_{k}\right)} 4\left(2 m(2 Z+1)+\varepsilon_{k}^{-1}\right)^{2} d \boldsymbol{x}\right. \\
\left.\quad+\int_{B_{\varepsilon_{k}}\left(\boldsymbol{R}_{k}\right)^{c}}\left[\frac{1}{\left|\boldsymbol{x}-\boldsymbol{R}_{k}\right|}-b\right]_{+}^{2} d \boldsymbol{x}-\frac{Z^{2} /(2 Z+1)^{2}}{16 m C_{\mathrm{A}}^{\prime} \nu^{2} D_{k}}\right)-(2 Z+1) b N
\end{gathered}
$$




$$
\begin{aligned}
& \geq-2 m C_{\mathrm{A}}^{\prime} \nu^{2}(2 Z+1)^{2} \sum_{k=1}^{K}\left(4\left(2 m(2 Z+1)+\varepsilon_{k}^{-1}\right)^{2} \pi \varepsilon_{k}^{2}\right. \\
& \left.\quad+2 \pi \int_{\varepsilon_{k}<r<b^{-1}}\left(r^{-1}-b\right)^{2} r d r-\frac{1}{144 m C_{\mathrm{A}}^{\prime} \nu^{2} D_{k}}\right)-(2 Z+1) b N \\
& \geq-4 \pi m C_{\mathrm{A}}^{\prime} \nu^{2}(2 Z+1)^{2} \sum_{k=1}^{K}\left(4+16 \frac{m^{2}(2 Z+1)^{2}}{b^{2}}\left(\varepsilon_{k} b\right)^{2}\right. \\
& \left.\quad+\left[-\frac{1}{2}\left(\varepsilon_{k} b\right)^{2}+2 \varepsilon_{k} b-\ln \varepsilon_{k} b-\frac{3}{2}\right]_{\varepsilon_{k} b<1}-\frac{b}{288 \pi m C_{\mathrm{A}}^{\prime} \nu^{2}}\left(D_{k} b\right)^{-1}\right) \\
& \quad-(2 Z+1) b N .
\end{aligned}
$$

Finally, taking $\varepsilon_{k}:=\min \left\{b^{-1}, D_{k}\right\}$ and in the latter case using that

$$
-\ln x-\frac{c}{x} \leq[-\ln c-1]_{+}
$$

for $0<x \leq 1$ and $c>0$, we have the further bound

$$
\begin{aligned}
& \geq-4 \pi m C_{\mathrm{A}}^{\prime} \nu^{2}(2 Z+1)^{2} K(\left.5+16 \frac{m^{2}(2 Z+1)^{2}}{b^{2}}+\left[\ln \frac{288 \pi m C_{\mathrm{A}}^{\prime} \nu^{2}}{b}\right]_{+}\right) \\
&-(2 Z+1) b N \\
& \geq-C \nu^{2} m(2 Z+1)^{2}(K+N),
\end{aligned}
$$

for some $C>0$, where in the last step we chose $b:=\nu^{2} m(2 Z+1)$.

\subsection{External potentials in the Calogero-Sutherland case}

Let us end with also considering an application of the above local exclusion and Lieb-Thirring inequalities for one dimensional statistics in the CalogeroSutherland case. We think of $N$ identical such particles placed in an external confining potential $V$, and would like to obtain a lower bound for the ground state energy $E_{0}$. We have from Theorem 18 that for $\alpha \geq 1$

$$
\begin{aligned}
& E_{0}:=\inf \left\{\int_{\mathbb{R}^{N}}\left(T_{\mathrm{CS}}^{N}(\psi ; \mathrm{x})+\sum_{j=1}^{N} V\left(x_{j}\right)|\psi|^{2}\right) d \mathrm{x}: \psi \in \mathscr{D}_{\mathrm{CS}}^{\alpha},\|\psi\|=1\right\} \\
& \geq \inf \left\{\int_{\mathbb{R}}\left(C_{\mathrm{CS}} \xi_{\mathrm{CS}}(\alpha)^{2} \tilde{\rho}(x)^{3}+V(x) \rho(x)\right) d x: \rho: \mathbb{R} \rightarrow \mathbb{R}_{\geq 0}, \int_{\mathbb{R}} \rho=N\right\} .
\end{aligned}
$$

Because of the local approximation $\tilde{\rho}$ to the density, this minimization problem is less tractable than e.g. the anyonic case in Section 6.1. However, what we can do instead is to consider a partition $\mathcal{P}$ of the real line into a collection 
of finite subintervals $\left\{I_{j}\right\}_{j=1}^{M}$ and an exterior domain $I_{\text {ext }}$, and on each such interval apply Lemma 12 or the local form of Theorem 18 ,

$$
\begin{aligned}
T_{\mathrm{CS}}+\int_{\mathbb{R}} V \rho \geq \sum_{j=1}^{M} & \left(T_{\mathrm{CS}}^{I_{j}}+\int_{I_{j}} V \rho\right)+\int_{I_{\mathrm{ext}}} V \rho \\
& \geq \sum_{j=1}^{M}\left(\frac{\xi_{\mathrm{CS}}(\alpha)^{2}}{\left|I_{j}\right|^{2}} \mathcal{E}\left(\int_{I_{j}} \rho\right)+V_{j} \int_{I_{j}} \rho\right)+V_{\mathrm{ext}} \int_{I_{\mathrm{ext}}} \rho,
\end{aligned}
$$

where we define $V_{j}:=\inf _{I_{j}} V$ and

$$
\mathcal{E}\left(\rho_{j}\right):=\max \left\{0, \rho_{j}-1, \chi_{\left\{\rho_{j} \geq 2\right\}} \rho_{j}^{3} / 32\right\}, \quad \rho_{j}:=\int_{I_{j}} \rho .
$$

Here we used (48) and (71) with $C_{\mathrm{CS}} \geq 1 / 32$, and note that $\rho_{j}-1 \leq \rho_{j}^{3} / 32$ and $\rho_{j} \geq 2$ implies $\rho_{j} \geq \rho_{c}$, with the critical point $\rho_{c} \approx 5.068>2$. We have hence reduced to a finite-dimensional minimization problem

$$
\begin{aligned}
E_{0} \geq E[\mathcal{P}]:= & \inf \left\{\mathcal{E}_{\mathcal{P}}[\boldsymbol{\rho}]: \boldsymbol{\rho}=\left(\left(\rho_{j}\right), \rho_{\mathrm{ext}}\right) \in \mathbb{R}_{\geq 0}^{M+1}, \sum_{j} \rho_{j}+\rho_{\mathrm{ext}}=N\right\}, \\
& \mathcal{E}_{\mathcal{P}}[\boldsymbol{\rho}]:=\sum_{j=1}^{M}\left(\frac{\xi_{\mathrm{CS}}(\alpha)^{2}}{\left|I_{j}\right|^{2}} \mathcal{E}\left(\rho_{j}\right)+V_{j} \rho_{j}\right)+V_{\text {ext }} \rho_{\text {ext }},
\end{aligned}
$$

for which a minimizer $\boldsymbol{\rho}$ exists due to the convexity of $\mathcal{E}_{\mathcal{P}}$. Depending on $N$ and the details of $V$, a hopefully good lower bound for $E_{0}$ (and an estimate for the ground state density $\rho$ ) can then be found by maximizing over all suitable partitions $\mathcal{P}$,

$$
E_{0} \geq \sup \left\{E[\mathcal{P}]: \mathcal{P}=\left(\left(I_{j}\right)_{j=1}^{M}, I_{\text {ext }}\right) \text { partition of } \mathbb{R}, M \in \mathbb{N}\right\} .
$$

As an illustration of this procedure we can consider the following example, which in the harmonic case $V(x)=\frac{\omega^{2}}{2}|x|^{2}$ can be compared to the exact ground state energy for the corresponding Calogero-Sutherland model 8 , 59], $E_{0}=\frac{1}{2} \omega N(1+\alpha(N-1))$. We can also compare to the approximative Thomas-Fermi method discussed in [54, 55].

Proposition 23. Given an external confining potential $V(x)=c^{\mu}|x|^{\mu}$, with $c, \mu>0$, and assuming $N \gg\left(\xi_{\mathrm{CS}}(\alpha) c\right)^{2 / \mu}$ and $\xi_{\mathrm{CS}}(\alpha) c N \gg 1$, we have for the ground state energy of $H=\hat{T}_{\mathrm{CS}}+\sum_{j=1}^{N} V\left(x_{j}\right)$

$$
E_{0} \geq C(\mu, N)\left(\xi_{\mathrm{CS}}(\alpha) c\right)^{\frac{2 \mu}{\mu+2}} N^{\frac{3 \mu+2}{\mu+2}}
$$

where $C(\mu, N) \geq 0$ is a constant s.t.

$$
\lim _{N \rightarrow \infty} C(\mu, N)=\left(\frac{\sqrt{3}}{4 \sqrt{2 \pi}}\right)^{\frac{2 \mu}{\mu+2}} \frac{\mu+2}{2 \mu^{2}} \frac{\Gamma\left(\frac{1}{\mu}\right)}{\Gamma\left(\frac{5}{2}+\frac{1}{\mu}\right)}\left(\frac{\Gamma\left(\frac{3}{2}+\frac{1}{\mu}\right)}{\Gamma\left(1+\frac{1}{\mu}\right)}\right)^{\frac{3 \mu+2}{\mu+2}} .
$$


In particular, $\liminf \operatorname{in}_{N \rightarrow \infty} E_{0} / N^{2} \geq \frac{\sqrt{3}}{8 \pi} \xi_{\mathrm{CS}}(\alpha) \omega$ for $\mu=2$ and $c=\omega / \sqrt{2}$.

Proof. Consider a simple partition of the form

$$
\mathcal{P}=\left([-(k+1) a,-k a]_{k=0}^{M-1},[k a,(k+1) a]_{k=0}^{M-1},(-\infty,-M a] \cup[M a,+\infty)\right),
$$

$a>0$, for which we have (making use of the symmetry, and with a suitable relabeling of $\rho_{j}$ )

$$
\mathcal{E}_{\mathcal{P}}[\boldsymbol{\rho}]=2 \sum_{k=0}^{M-1}\left(\frac{\xi_{\mathrm{CS}}(\alpha)^{2}}{a^{2}} \mathcal{E}\left(\rho_{k}\right)+c^{\mu}(k a)^{\mu} \rho_{k}\right)+c^{\mu}(M a)^{\mu} \rho_{\mathrm{ext}} .
$$

We extremize the functional

$$
\begin{aligned}
& F[\boldsymbol{\rho}, \lambda]:=\mathcal{E}_{\mathcal{P}}[\boldsymbol{\rho}]-\lambda\left(\sum_{j} \rho_{j}+\rho_{\mathrm{ext}}\right)+\lambda N \\
& =2 \sum_{k=0}^{M-1}\left(\frac{\xi_{\mathrm{CS}}(\alpha)^{2}}{a^{2}} \mathcal{E}\left(\rho_{k}\right)+\left(c^{\mu} a^{\mu} k^{\mu}-\lambda\right) \rho_{k}\right)+\left(c^{\mu} a^{\mu} M^{\mu}-\lambda\right) \rho_{\mathrm{ext}}+\lambda N
\end{aligned}
$$

to find, if $M$ is chosen large enough, $\rho_{\text {ext }}=0$ and $\rho_{k}=0$ for $c^{\mu} a^{\mu} k^{\mu}-\lambda \geq 0$, $\rho_{k}=1$ for $0<\lambda-c^{\mu} a^{\mu} k^{\mu}<\xi_{\mathrm{CS}}(\alpha)^{2} / a^{2}$, and otherwise

$$
\rho_{k}=\max \left\{\rho_{c}, \frac{a \sqrt{32 / 3}}{\xi_{\mathrm{CS}}(\alpha)} \sqrt{\lambda-c^{\mu} a^{\mu} k^{\mu}}\right\} .
$$

Hence, $N=2 \sum_{k} \rho_{k}+\rho_{\text {ext }}=$

$$
\begin{gathered}
=\sum_{\substack{k \geq 0 \text { s.t. } \\
\lambda-c^{\mu} a^{\mu} k^{\mu} \geq \xi_{\mathrm{CS}}(\alpha)^{2} / a^{2}}} 2 \max \left\{\rho_{c}, \frac{a \sqrt{32 / 3}}{\xi_{\mathrm{CS}}(\alpha)} \sqrt{\lambda-c^{\mu} a^{\mu} k^{\mu}}\right\} \\
+\sum_{\substack{k \geq 0 \text { s.t. } \\
0<\lambda-c^{\mu} a^{\mu} k^{\mu}<\xi_{\mathrm{CS}}(\alpha)^{2} / a^{2}}} 2 .
\end{gathered}
$$

Now, let $a:=1 / c$ and assume that $N \gg(\xi c)^{2 / \mu}$ and $\xi c N \gg 1$. Then

$$
\begin{aligned}
& \xi c N \sim \sum_{\begin{array}{r}
k \geq 0 \text { s.t. } \\
\lambda-k^{\mu} \geq \xi^{2} c^{2}
\end{array}} 2 \sqrt{32 / 3} \sqrt{\lambda-k^{\mu}} \\
& \sim 8 \sqrt{2 / 3} \int_{0}^{\lambda^{1 / \mu}} \sqrt{\lambda-k^{\mu}} d k=8 \sqrt{2 / 3} I(\mu) \lambda^{1 / 2+1 / \mu},
\end{aligned}
$$

where

$$
I(\mu):=\int_{0}^{1} \sqrt{1-x^{\mu}} d x=\frac{\sqrt{\pi}}{2} \frac{\Gamma\left(1+\frac{1}{\mu}\right)}{\Gamma\left(\frac{3}{2}+\frac{1}{\mu}\right)} .
$$




$$
\begin{aligned}
& \text { Hence, } \lambda \sim\left(\frac{\sqrt{3 / 2}}{8 I(\mu)} \xi c N\right)^{\frac{2 \mu}{\mu+2}} \text { and } \\
& \begin{aligned}
E_{0} \geq \mathcal{E}_{\mathcal{P}}[\boldsymbol{\rho}] \\
\geq 2 \sum_{\substack{k \geq 0 \text { s.t. } \\
\lambda-k^{\mu} \geq \xi^{2} c^{2}}}\left(\frac{\xi^{2} c^{2}}{32}\left(\frac{\sqrt{32 / 3}}{\xi c} \sqrt{\lambda-k^{\mu}}\right)^{3}+k^{\mu}\left(\frac{\sqrt{32 / 3}}{\xi c} \sqrt{\lambda-k^{\mu}}\right)\right) \\
\quad=\frac{8 \sqrt{2 / 3}}{\xi c} \sum_{k}\left(\frac{1}{3}\left(\lambda-k^{\mu}\right)^{\frac{3}{2}}+k^{\mu} \sqrt{\lambda-k^{\mu}}\right) \sim 8 \sqrt{2 / 3} J(\mu) \frac{\lambda^{\frac{3}{2}+\frac{1}{\mu}}}{\xi c},
\end{aligned}
\end{aligned}
$$

where

$$
J(\mu):=\frac{1}{3} \int_{0}^{1}\left(1-x^{\mu}\right)^{\frac{3}{2}} d x+\int_{0}^{1} x^{\mu} \sqrt{1-x^{\mu}} d x=\frac{\sqrt{\pi}}{2} \frac{\mu+2}{2 \mu^{2}} \frac{\Gamma\left(\frac{1}{\mu}\right)}{\Gamma\left(\frac{5}{2}+\frac{1}{\mu}\right)} .
$$

It follows under the above conditions that

$$
\liminf _{N \rightarrow \infty} \frac{E_{0}}{N^{\frac{3 \mu+2}{\mu+2}}} \geq(8 \sqrt{2 / 3})^{-\frac{2 \mu}{\mu+2}} \frac{J(\mu)}{I(\mu)^{\frac{3 \mu+2}{\mu+2}}}(\xi c)^{\frac{3 \mu+2}{\mu+2}-1},
$$

which is the asymptotics of the r.h.s. of (86).

\section{References}

[1] C. Aneziris, A. P. Balachandran, D. Sen, Statistics in one dimension, Int. J. Mod. Phys. A 6 (1991) 4721-4751.

[2] D. Arovas, J. R. Schrieffer, F. Wilczek, Fractional Statistics and the Quantum Hall Effect, Phys. Rev. Lett. 53 (1984) 722-723.

[3] G. A. Baker, G. S. Canright, S. B. Mulay, C. Sundberg, On the Spectral Problem for Anyons, Commun. Math. Phys. 153 (1993) 277-295.

[4] B. Basu-Mallick, P. K. Ghosh, K. S. Gupta, Inequivalent quantizations of the rational Calogero model, Phys. Lett. A 311 (2003) 87-92.

[5] R. K. Bhaduri, G. Date, M. V. N. Murthy, J. Law, On a class of noninterpolating solutions of the many-anyon problem, J. Phys. A: Math. Gen. 25 (1992) 6163-6168.

[6] I. Bloch, J. Dalibard, W. Zwerger, Many-body physics with ultracold gases, Rev. Mod. Phys. 80 (2008) 885-964.

[7] L. Bruneau, J. Derezinski, V. Georgescu, Homogeneous Schrödinger operators on half-line, Ann. Henri Poincaré 12 (2011) 547-590. 
[8] F. Calogero, Ground State of a One-Dimensional N-Body System, J. Math. Phys. 10 (1969) 2197-2200.

[9] R. Chitra, D. Sen, Ground state of many anyons in a harmonic potential, Phys. Rev. B 46 (1992) 10923-10930.

[10] C. Chou, Multianyon spectra and wavefunctions, Phys. Rev. D 44 (1991) 2533-2547.

[11] G. Dell'Antonio, R. Figari, A. Teta, Statistics in Space Dimension Two, Lett. Math. Phys. 40 (1997) 235-256.

[12] J. Dolbeault, A. Laptev, M. Loss, Lieb-Thirring inequalities with improved constants, J. Eur. Math. Soc. 10 (2008) 1121-1126.

[13] F. J. Dyson, A. Lenard, Stability of Matter. I, J. Math. Phys. 8 (1967) 423-434.

[14] F. J. Dyson, Stability of Matter, in Statistical Physics, Phase Transitions and Superfluidity, Brandeis University Summer Institute in Theoretical Physics 1966, pp. 179-239, (Gordon and Breach Publishers, New York, 1968).

[15] L. Erdős, V. Vougalter, Pauli Operator and Aharonov-Casher Theorem for Measure Valued Magnetic Fields, Comm. Math. Phys. 225 (2002) 399-421.

[16] L. Fehér, I. Tsutsui, T. Fülöp Inequivalent quantizations of the threeparticle Calogero model constructed by separation of variables, Nucl. Phys. B 715 (2005) 713-757.

[17] R. L. Frank, R. Seiringer, Lieb-Thirring Inequality for a Model of Particles with Point Interactions, J. Math. Phys. 53, 095201 (2012).

[18] J. Fröhlich, Quantum statistics and locality, in Proceedings of the Gibbs Symposium (New Haven, CT, 1989), pp. 89-142, Amer. Math. Soc., Providence, RI, 1990.

[19] G. Gentile, Osservazioni sopra le statistiche intermedie, Il Nuovo Cimento 17 (1940) 493-497.

[20] G. Gentile, Le statistiche intermedie e le proprieta dell'elio liquido, Il Nuovo Cimento 19 (1942) 109-125.

[21] G. A. Goldin, S. Majid, On the Fock space for nonrelativistic anyon fields and braided tensor products, J. Math. Phys. 45 (2004) 3770-3787.

[22] G. A. Goldin, R. Menikoff, D. H. Sharp, Representations of a local current algebra in nonsimply connected space and the Aharonov-Bohm effect, J. Math. Phys. 22 (1981) 1664-1668. 
[23] F. D. M. Haldane, "Fractional statistics" in Arbitrary Dimensions: A Generalization of the Pauli Principle, Phys. Rev. Lett. 67 (1991) 937940 .

[24] S. B. Isakov, Statistics in one dimension: Heisenberg and Schrödinger quantizations, Mod. Phys. Lett. A 7 (1992) 3045-3051.

[25] S. B. Isakov, Statistical Mechanics for a class of Quantum Statistics, Phys. Rev. Lett. 73 (1994) 2150-2153.

[26] A. Khare, Fractional Statistics and Quantum Theory, (World Scientific, Singapore, Second Edition 2005).

[27] T. Kinoshita, T. Wenger, D. S. Weiss, Observation of a OneDimensional Tonks-Girardeau Gas, Science 305 (2004) 1125-1128.

[28] R. B. Laughlin, Nobel Lecture: Fractional quantization, Rev. Mod. Phys. 71 (1999) 863-874.

[29] J. M. Leinaas, J. Myrheim, On the Theory of Identical Particles, Il Nuovo Cimento 37B (1977) 1-23.

[30] J. M. Leinaas, J. Myrheim, Intermediate statistics for vortices in superfiuid films, Phys. Rev. B 37 (1988) 9286-9291.

[31] J. M. Leinaas, J. Myrheim, Heisenberg quantization for systems of identical particles, Int. J. Mod. Phys. A 8 (1993) 3649-3695.

[32] A. Lenard, Lectures on the Coulomb Stability Problem, in Statistical mechanics and mathematical problems, Battelle Rencontres, Seattle, Wash., 1971, Lect. Notes Phys., Vol. 20, pp. 114-135, 1973.

[33] A. Lerda, Anyons, (Springer-Verlag, Berlin-Heidelberg, 1992).

[34] E. H. Lieb, W. Liniger, Exact Analysis of an Interacting Bose Gas. I. The General Solution and the Ground State, Phys. Rev. 130 (1963) 1605-1616.

[35] E. H. Lieb, M. Loss, Analysis, Second Edition, (American Mathematical Society, Providence, RI, 2001).

[36] E. H. Lieb, R. Seiringer, J. Yngvason, One-Dimensional Bosons in Three-Dimensional Traps, Phys. Rev. Lett. 91 (2003) 150401.

[37] E. H. Lieb, R. Seiringer, The stability of matter in quantum mechanics, (Cambridge University Press, Cambridge, 2010).

[38] E. H. Lieb, W. Thirring, Bound for the Kinetic Energy of Fermions which Proves the Stability of Matter, Phys. Rev. Lett. 35 (1975) 687689 . 
[39] E. H. Lieb, W. Thirring, Inequalities for the Moments of the Eigenvalues of the Schrödinger Hamiltonian and Their Relation to Sobolev Inequalities, in Studies in Mathematical Physics, pp. 269-303, Princeton University Press, 1976.

[40] D. Loss, Y. Fu, Second Virial Coefficient of an Interacting Anyon Gas, Phys. Rev. Lett. 67 (1991) 294-297.

[41] D. Lundholm, Geometric extensions of many-particle Hardy inequalities, arXiv:1101.2653.

[42] D. Lundholm, J. P. Solovej, Hardy and Lieb-Thirring inequalities for anyons, Commun. Math. Phys. 322 (2013) 883-908.

[43] D. Lundholm, J. P. Solovej, Local exclusion for intermediate and fractional statistics, arXiv:1205.2520.

[44] M. V. N. Murthy, J. Law, M. Brack, R. K. Bhaduri, Quantum Spectrum of Three Anyons in an Oscillator Potential, Phys. Rev. Lett. 67 (1991) 1817-1820.

[45] J. Myrheim, Anyons, in Topological aspects of low dimensional systems (Les Houches, 1998), pp. 265-413, EDP Sci., Les Ulis, 1999.

[46] S. Ouvry, Anyons and Lowest Landau Level Anyons, Séminaire Poincaré XI (2007) 77-107.

[47] B. Paredes et al., Tonks-Girardeau gas of ultracold atoms in an optical lattice, Nature 429 (2004) 277-281.

[48] A. P. Polychronakos, Non-relativistic bosonization and fractional statistics, Nucl. Phys. B 324 (1989) 597-622.

[49] A. P. Polychronakos, Exchange Operator Formalism for Integrable Systems of Particles, Phys. Rev. Lett. 69 (1992) 703-705.

[50] A. P. Polychronakos, Generalized statistics in one dimension, in Topological aspects of low dimensional systems (Les Houches, 1998), pp. 415-471, EDP Sci., Les Ulis, 1999.

[51] R. E. Prange, S. M. Girvin (eds.), The Quantum Hall Effect, (SpringerVerlag, Second Edition 1990).

[52] M. Rumin, Balanced distribution-energy inequalities and related entropy bounds, Duke Math. J. 160 (2011) 567-597.

[53] D. Sen, Some supersymmetric features in the spectrum of anyons in a harmonic potential, Phys. Rev. D 46 (1992) 1846-1857. 
[54] D. Sen, R. K. Bhaduri, Thomas-Fermi Method for Particles Obeying Generalized Exclusion Statistics, Phys. Rev. Lett. 74 (1995) 3912-3915.

[55] A. Smerzi, Quantum Corrections to the Thomas-Fermi Theory for Fractional-Statistics Particles, Phys. Rev. Lett. 76 (1996) 559-562.

[56] M. Sporre, J. J. M. Verbaarschot, I. Zahed, Numerical Solution of the Three-Anyon Problem, Phys. Rev. Lett. 67 (1991) 1813-1816.

[57] M. Sporre, J. J. M. Verbaarschot, I. Zahed, Four anyons in a harmonic well, Phys. Rev. B. 46 (1992) 5738-5741.

[58] R. F. Streater, I. F. Wilde, Fermion states of a boson field, Nucl. Phys. B 24 (1970) 561-575.

[59] B. Sutherland, Quantum Many-Body Problem in One Dimension: Ground State, J. Math. Phys. 12 (1971) 246-250.

[60] F. Wilczek, Magnetic Flux, Angular Momentum, and Statistics, Phys. Rev. Lett 48 (1982) 1144-1146; Quantum Mechanics of Fractional-Spin Particles, Phys. Rev. Lett 49 (1982) 957-959.

[61] F. Wilczek, Fractional Statistics and Anyon Superconductivity, (World Scientific, Singapore, 1990).

[62] Y-S. Wu, Multiparticle Quantum Mechanics Obeying Fractional Statistics, Phys. Rev. Lett. 53 (1984) 111-114. 